\title{
Modelling the Inflation and Elastic Instabilities of Rubber-Like Spherical and Cylindrical Shells Using a New Generalised Neo-Hookean Strain Energy Function
}

\author{
Afshin Anssari-Benam ${ }^{1}$ (D) Andrea Bucchi ${ }^{1}$. \\ Giuseppe Saccomandi ${ }^{2,3}$
}

Dedicated to Millard, an esteemed scholar and a gentleman

Received: 8 September 2020 / Accepted: 7 March 2021 / Published online: 15 April 2021

(C) The Author(s) 2021

\begin{abstract}
The application of a newly proposed generalised neo-Hookean strain energy function to the inflation of incompressible rubber-like spherical and cylindrical shells is demonstrated in this paper. The pressure $(P)$ - inflation ( $\lambda$ or $v)$ relationships are derived and presented for four shells: thin- and thick-walled spherical balloons, and thin- and thick-walled cylindrical tubes. Characteristics of the inflation curves predicted by the model for the four considered shells are analysed and the critical values of the model parameters for exhibiting the limit-point instability are established. The application of the model to extant experimental datasets procured from studies across 19th to 21 st century will be demonstrated, showing favourable agreement between the model and the experimental data. The capability of the model to capture the two characteristic instability phenomena in the inflation of rubber-like materials, namely the limit-point and inflation-jump instabilities, will be made evident from both the theoretical analysis and curve-fitting approaches presented in this study. A comparison with the predictions of the Gent model for the considered data is also demonstrated and is shown that our presented model provides improved fits. Given the simplicity of the model, its ability to fit a wide range of experimental data and capture both limit-point and inflationjump instabilities, we propose the application of our model to the inflation of rubber-like materials.
\end{abstract}

Mathematics Subject Classification $70 \mathrm{~K} 50 \cdot 74 \mathrm{~A} 20 \cdot 74 \mathrm{~B} 20$

Keywords Inflation · Rubber-like materials · Spherical and cylindrical shells · Instability · Modelling

A. Anssari-Benam

afshin.anssari-benam@port.ac.uk

1 Cardiovascular Engineering Research Lab (CERL), School of Mechanical and Design Engineering, University of Portsmouth, Anglesea Road, Portsmouth PO1 3DJ, UK

2 Dipartimento di Ingegneria, Università degli studi di Perugia, Via G. Duranti, Perugia 06125, Italy

3 School of Mathematics, Statistics and Applied Mathematics, NUI Galway, University Road, Galway, Ireland 
Fig. 1 A typical inflation pressure $(P)$ versus stretch $(\lambda)$ or volume $(v)$ curve for a spherical/cylindrical rubber shell, exhibiting limit-point and inflation-jump instabilities

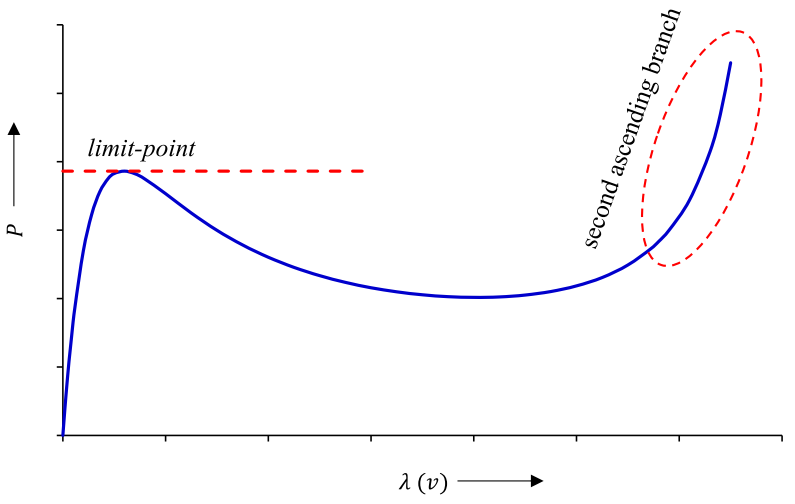

\section{Introduction}

The inflation of incompressible isotropic rubber-like spherical and cylindrical shells has been well codified using the nonlinear theory of elasticity. Upon selecting an appropriate form of a strain energy function $W$ and imposing the correct boundary conditions, solutions may be obtained of analytical relationships between the inflation pressure $P$ and the deformation stretch $\lambda$ (or volume $v$ ). The physics of inflation of rubber shells, however, evinces interesting instabilities. Adopting the same terminology as advocated by Mangan and Destrade [27], the pressure-deformation curve in spherical and cylindrical rubber shells quickly reaches a maximum in pressure, which is referred to as the limit-point instability. Upon further inflation, the pressure decreases but then increases rapidly again until the bursting point. In practice, if we keep inflating after the maximum pressure limit-point instability, the stretch suddenly jumps to a larger value on the second ascending branch, which is called inflation-jump instability. A representative illustration of these features is shown in Fig. 1.

The inflation of rubber balloons is considered an archetypal problem in the mathematical modelling of nonlinear elasticity, and inflation experiments are relatively straightforward to perform compared with other more intricate deformation experiments such as shear or torsion. The solution of the inflation problem for an arbitrary strain energy function $W$ may be traced back first to the work of Green and Zerna in the limit of the thin shell approximation [17]. Similar results were later derived by Green and Adkins from a general theory of incompressible, hyperelastic membranes [16], and by Beatty from a purely energetic consideration [5]. See also the book by Müller and Strehlow for a thermodynamics approach to the problem of inflation [29]. Beatty's work, however, contains a detailed analysis of the inflation problem using the Mooney-Rivlin, neo-Hookean and Fung-Demiray incompressible strain energy functions and the compressible Blatz-Ko material. By comparing the predictions of the mathematical models with the experimental data, Beatty arrived at the following fundamental conclusions in relation to the inflation problem within the framework of nonlinear elasticity [5]:

(i) The experimental results on the inflation of rubber balloons are consistent with the assumption of incompressibility;

(ii) While the Mullin's effect can affect the quality of the experimental data, a careful preconditioning of the specimens allows the study of inflation within the framework of hyperelasticity with a good approximation;

(iii) Of the considered strain energy functions, the Mooney-Rivlin model which includes the $I_{2}$ invariant may be a more appropriate mathematical model; and 
(iv) The ultimate stiffening effect as a result of the influence of the molecular chain structure is a prominent characteristic in the balloon inflation problem.

At first glance, these results may indicate that any incompressible, hyperelastic strain energy function with an $I_{2}$ term may be an appropriate choice for modelling the true physics of the inflation phenomenon. Interestingly, however, not many strain energy functions $W$ in the literature are capable of reproducing and capturing the aforementioned instabilities, and their application to modelling the large deformation of rubber-like materials may prove specious. As shown in [27], a long list of generalised neo-Hookean strain energy densities and other types of functions including those embodying $I_{2}$ terms either fail to reproduce the limit-point instability or the ascending branch to capture the inflation-jump instability. These include the neo-Hookean, Varga, Dickie-Smith (see [11] for this model), Gent-Thomas (see [15]), one-term Ogden and Mooney (of 3rd degree) models. Even the Mooney-Rivlin model, while capable of predicting the instabilities, fails to accurately capture the "the ultimate stiffening effect", i.e., point (iv) of Beatty's main findings summarised earlier in the foregoing, and therefore does not provide a good fit to the second ascending branch of the inflation curve (see [27]). Indeed, a primary finding of Mangan and Destrade was that perhaps only the Gent-Gent model, i.e., the modification adduced by Pucci and Saccomandi [32] to the original Gent model [12], may be the most versatile for capturing the true physics of inflation of rubber-like balloon shells [27]. We note here another important contribution in the study of inflation instabilities by Kanner and Horgan [23] using limiting chain extensibility models, further underlining the ability of such models and in particular that of the Gent in capturing the inflation behaviour of rubber-like thin shells. These results therefore appear to indicate that the generalised neo-Hookean part (the $I_{1}$ term) of the model may be more prominent in predicting the true inflation behaviour of rubber shells, and the role of the $I_{2}$ term may be less critical. Note that a similar sentiment in relation to the role of $I_{2}$ in modelling the biomechanics of soft tissues has also been echoed by Ogden and Saccomandi [30]. For a recent discussion and summary on the role of the $I_{2}$ invariant in modelling the deformation of rubber-like materials see the work of Destrade et al. [10].

Generalised neo-Hookean strain energy functions that have their roots in the statistical mechanics of molecular chains in rubber elasticity enjoy the advantage of incorporating the (meso)structural features of the material. One such structural feature is the chain extensibility limit, which gives rise to the strain hardening effect observed in the defamation of rubber-like materials. See also [30] in the context of the biomechanics of soft tissues. One of the celebrated generalised neo-Hookean functions in this regard is the Gent model. Indeed, using this model the study of Mangan and Destrade [27] underlines a fundamental result in addressing point (iv) of Beatty's main findings, i.e., modelling the "ultimate stiffening effect". However, the focus of that study was the Gent-Gent model and how it compares with the Mooney-Rivlin model in capturing the inflation phenomenon in rubber-like materials. The problem of inflation, however, is not limited to the scope of application of the Gent-Gent model and further improvements in modelling results and analysis may still be achieved, as we endeavour to demonstrate in this contribution. One way of achieving this improvement is to devise a more refined model than that of the Gent.

The aim of this paper is to consider the foregoing problem of modelling the inflation and the ensuing instabilities of rubber-like shells within the framework of a new generalised neo-Hookean strain energy function from the family of limiting chain extensibility models in the form of [3]:

$$
W=\mu N\left[\frac{1}{6 N}\left(I_{1}-3\right)-\ln \left(\frac{I_{1}-3 N}{3-3 N}\right)\right],
$$


where $\mu$ is a measure of shear modulus, $N$ is the classical number of Kuhn segments, and $I_{1}$ is the first principal invariant of the left Cauchy-Green tensor B. This model is a reformulation of that proposed previously for application within the context of soft tissue biomechanics [2]. Note that $\mu$ in the above model is not the infinitesimal shear modulus $\mu_{0}$. On using $\mu_{0}=2\left(\frac{\partial W}{\partial I_{1}}\right)_{I_{1}=3}$ (see, e.g., [10]), the following relationship between $\mu$ in our model and the infinitesimal shear modulus $\mu_{0}$ is rendered:

$$
\frac{\partial W}{\partial I_{1}}=\mu N\left[\frac{1}{6 N}-\frac{1}{I_{1}-3 N}\right] \Rightarrow \mu_{0}=2 \mu N\left[\frac{1}{6 N}-\frac{1}{3-3 N}\right] \Rightarrow \mu=\mu_{0}\left[\frac{3-3 N}{1-3 N}\right] .
$$

The interest in the application of this model may be manifold. First, the model is directly derived from the molecular theory of rubbers and therefore the model parameters have physical objectivity and meaning (see [2] and [3] for a detailed derivation). The Gent model, by contrast, was intuitively derived to mimic the strain hardening effects of the statistical molecular chain models involving the inverse Langevin function, in a purely phenomenological way [33]. Indeed, the Gent model is the simplest model of a class of models that generalise Rivlin's approach of deriving analytical strain-energy functions using rational function approximants [20]. In this sense, the proposed model is structurally a refinement of the celebrated Gent model. Second, the model has a simple mathematical form and only embodies two parameters. Third, it is able to provide improved fits to a wide range of pure homogenous and simple shear deformation data (see [3] for more details and applications), and as will be shown further on also to many inflation datasets, with respect to other similar models such as that of the Gent. Accordingly, the proposed model provides a more accurate tool for investigating and modelling Beatty's "ultimate stiffening effect as a result of the influence of the molecular chain structure in the balloon inflation problem". Our analysis in this work is therefore not just contrasting a model against extant experimental data, but rather is to offer new insight into a more accurate modelling of the "ultimate stiffening effect" and the instabilities inherent to the inflation behaviour of rubber-like materials. It is only by this approach, i.e., devising and offering more accurate modelling tools, that a meaningful investigation of other and more general issues such as the significance of the $I_{2}$ term in modelling the inflation phenomenon may be carried out and accomplished.

In view of the shortcomings of a body of the existing models in the literature to capture the inflation of rubber-like shells recounted in the foregoing, and the aforementioned advantages of the proposed model in Eq. (1), it is our aim in this paper to show the application of this model to the inflation problem of rubber-like shells and demonstrate its capability in capturing the two characteristic instability phenomena of the inflation problem. This paper, therefore, is first concerned with deriving the equations for inflation pressure as a function of the amount of inflation, i.e., either the stretch $\lambda$ or volume $v$, using the proposed model for four rubber shells: thin-wall and thick-wall spherical balloons, and thin-wall and thickwall cylindrical tubes. We then apply the model to a range of extant experimental data for comparing the model predictions versus the data and present the modelling results.

Of the four inflation problems that will be considered in this paper, the thin spherical balloon shell provides a simple context for the application of the presented model in the study of inflation within the theory of non-linear elasticity. It is well known that capturing the characteristics of the inflation curve crucially depends on the suitability of the considered model. For example, not all models are capable of capturing a single local maximum pressure, a monotonic stable inflation, or both the limit-point and inflation-jump instabilities (a classic example is the neo-Hookean strain energy function; however see [23] and [27] for more analysis). Therefore, while extensively studied, the inflation of a thin spherical balloon 
shell is an important first step for a convincing demonstration of the predictive capabilities of our model in capturing the true physics of inflation and the ensuing instabilities. We will then consider the problem of the inflation of a thick-walled spherical balloon. This problem has been less well studied; nevertheless we will devise and present the pressure-inflation relationships for thick-wall spherical balloons using our model. A particular field of application of thick-wall spherical shells may be in the study of (small) cavities; though we will not consider such studies in our current work. Next, we will analyse the inflation of thinand thick-walled cylindrical tubes. Comparatively, the inflation of cylindrical shells has received less attention than studying spherical balloon shells. However, a fortiori, analysing the inflation of cylindrical shells is of critical importance. First, all the intricacies of inflation characteristics and instabilities discussed in the case of spherical balloon shells may also be extended to cylindrical shells. Second, instabilities in the inflation of cylindrical tubes manifest themselves as non-uniform deformations along the tube, where a localised portion of the tube may experience a bulge while other parts of the tube may remain moderately inflated. This mode of inflation instability is reminiscent of arterial aneurysms, and hence appropriate modelling tools for the inflation of cylindrical shells may also prove insightful in studying the biomechanics of biological tissues in healthy and diseased conditions [6, 7]. As will be cast forth, our model predicts a stable monotonic inflation for a thick walled cylindrical tube, while the inflation of a thin wall cylinder exhibits both monotonic inflation and limitpoint and inflation-jump instabilities, depending on the value of the model parameter $N$. Therefore, based on the physics of the problem, appropriate analytical tools may be applied.

The derivation of pressure-inflation equations for all the four aforementioned cases will be presented in $\$ 2$. Theoretical analyses surrounding the characteristics of the pressure curves and critical values of the model parameters related to instabilities will also be presented for each case in this section. The application of the model to extant experimental datasets will be demonstrated in $\$ 3$ along with the modelling results. It will be shown how the model favourably captures the true physics of inflation for datasets which either exhibit the inflation-jump instability or do not. In addition, we highlight a number of interesting findings including an appreciable improvement of the fits with respect to the Gent model's predictions and that the inclusion of an $I_{2}$ term may not often result in an improved fit compared with the $I_{1}$-only function for this class of (inflation) deformation. Concluding remarks are conferred in $\S 4$.

\section{Model Formulation}

In this section we formulate and present the pressure-inflation relationships derived from our proposed model in Eq. (1) for spherical and cylindrical shells. The underlying assumption in deriving these relationships is that the Young-Laplace equation of equilibrium should apply. While we employ this assumption here $a$ priori, a rigorous derivation and justification for making this assumption has been presented in [27], starting from the exact solution for inflation of finite thickness non-linearly elastic shells.

\subsection{Pressure-Inflation Relationship for Thin Spherical Balloon Shells}

Starting from the Young-Laplace equation of equilibrium for bubbles, and noting that the Cauchy stress $\sigma$ is equal to the tension $T$ divided by the deformed thickness, the wellestablished relationship between the (Cauchy) stress and inflation pressure may be written 
as:

$$
\sigma=\frac{P r}{2 h}
$$

where $r$ and $h$ are the deformed radius and thickness, respectively. On assuming that the balloon remains spherical on inflation and due to the symmetry of the problem, a state of equi-biaxial extension is rendered where:

$$
\left\{\begin{array}{l}
\lambda_{1}=\lambda_{2}=\lambda=\frac{r}{r_{0}}, \\
\lambda_{3}=\lambda^{-2}=\frac{h}{h_{0}},
\end{array}\right.
$$

are the principal stretches under the assumption of incompressibility, and $r_{0}$ and $h_{0}$ are the undeformed radius and thickness, respectively. Given that the Cauchy stress tensor $\sigma$ under incompressibility is given as:

$$
\sigma=2 \frac{\partial W}{\partial I_{1}} \mathbf{B}-p \mathbf{I}
$$

with $\mathbf{B}=\mathbf{F F}^{\mathrm{T}}$ and $\mathbf{I}$ the identity matrix, Eq. (3) may be re-written to give an explicit relationship for the inflation pressure $P$ and inflation stretch $\lambda$ as:

$$
P=4 \frac{h_{0}}{r_{0}}\left(\lambda^{-1}-\lambda^{-7}\right) \frac{\partial W}{\partial I_{1}} .
$$

For the specific form of our proposed $W$ function in Eq. (1), where:

$$
\frac{\partial W}{\partial I_{1}}=\mu\left[\frac{I_{1}-9 N}{6\left(I_{1}-3 N\right)}\right],
$$

and $I_{1}=2 \lambda^{2}+\lambda^{-4}$, the final form of the inflation pressure - stretch relationship in our model for thin spherical shells may be arrived at as:

$$
P=\frac{2 h_{0}}{3 r_{0}} \mu\left(\lambda^{-1}-\lambda^{-7}\right)\left(\frac{2 \lambda^{2}+\lambda^{-4}-9 N}{2 \lambda^{2}+\lambda^{-4}-3 N}\right) .
$$

In some applications, it may prove more convenient to use an auxiliary variable $v=\lambda^{3}$ as a measure of volume expansion ratio. Using this new inflation measure, Eq. (8) may be simply re-written as:

$$
P=\frac{2 h_{0}}{3 r_{0}} \mu\left(v^{-\frac{1}{3}}-v^{-\frac{7}{3}}\right)\left(\frac{2 \sqrt[3]{v^{2}}+\sqrt[3]{v^{-4}}-9 N}{2 \sqrt[3]{v^{2}}+\sqrt[3]{v^{-4}}-3 N}\right)
$$

Due to the existence of a singularity point at a critical $\lambda$ or $v$ in our model (where the denominator of the argument in the second bracket of either Eqs. (8) or (9) will approach zero), the model will always predict an ascending branch a priori with increase in $\lambda$ or $v$. However, for the existence of the limit-point instability, a first point of maximum in the inflation pressure curve needs to exist which in turn requires that the model parameter $N$ to 


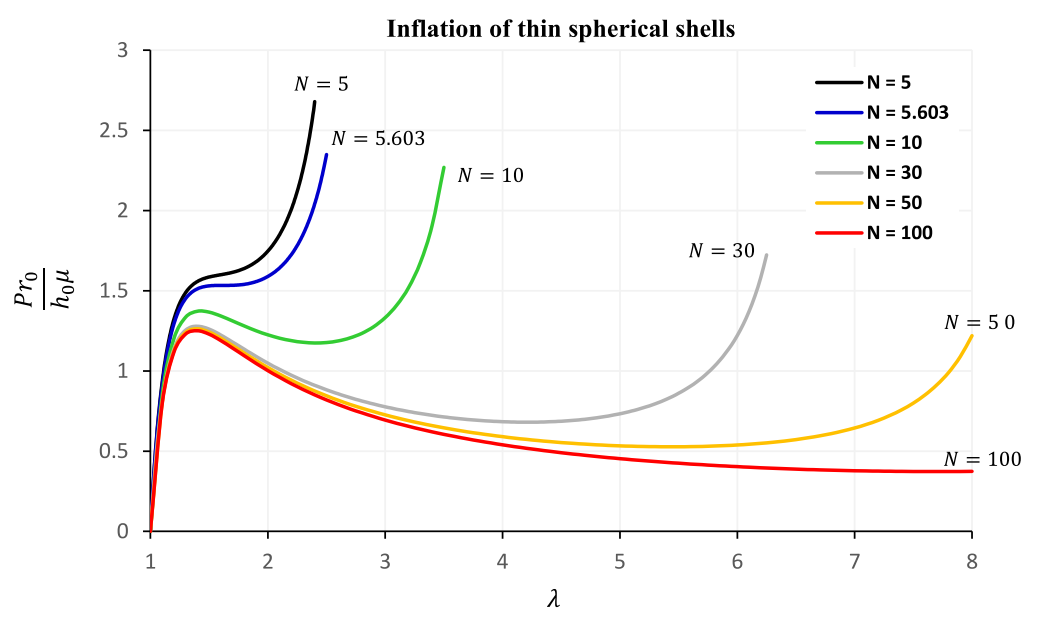

Fig. 2 Normalised inflation pressure versus stretch for a thin spherical balloon shell using our proposed model in Eq. (8) at various values of $N$. For $N<5.603$, it is clear that the inflation is monotonic and stable; while for $N>5.603$ both limit-point and inflation-jump instabilities are evident

fall within a certain range. In order to calculate the critical value of $N$ where the limit-point instability ceases to exist, we require using Eqs. (8) that [23, 27]:

$$
\frac{\mathrm{d} P}{\mathrm{~d} \lambda}=0, \quad \frac{\mathrm{d}^{2} P}{\mathrm{~d} \lambda^{2}}=0,
$$

or equivalently using Eq. (9) with respect to $v$. The ensuing expressions can be derived straightforwardly but are rather lengthy and we therefore refrain to reproduce them in full here in the interest of brevity. However, it can be shown that Eq. (10) holds if:

$$
-4 \lambda^{18}+48 N \lambda^{16}-27 N^{2} \lambda^{14}+24 \lambda^{12}-204 N \lambda^{10}+189 N^{2} \lambda^{8}+27 \lambda^{6}-60 N \lambda^{4}+7=0 .
$$

We obtain that Eq. (11) has two real roots in $\lambda>1$ if the value of $N$ is above a certain threshold, denoted by $N_{t}$, as:

$$
N>N_{t} \cong 5.603
$$

For $N<5.603$, Eqs. (8) and (9) predict a monotonic and stable inflation. For $N>5.603$, the inflation pressure curves will exhibit a maximum pressure, namely the limit-point instability, and the second ascending branch, namely the inflation-jump instability. Normalised inflation pressure curves produced by Eq. (8) using various values of $N$ below and above $N_{t}$ are presented in Fig. 2, reinforcing the existence of the threshold $N_{t}$ value.

\subsection{Pressure-Inflation Relationship for Thick Wall Spherical Balloon Shells}

We employ the approach devised by Gent in analysing the inflation of thick-walled spherical balloons [13], with a more detailed derivation of the relationships. In this regard, the internal pressure $P$ required to inflate a thick-walled spherical shell may be obtained by integrating the contribution of $\mathrm{d} P$ from concentric thin-walled balloon shells with wall thickness $\mathrm{d} r_{0}$. 
The relationship between the (Cauchy) stress and inflation pressure in Eq. (3) may therefore be adjusted as:

$$
\sigma=\frac{\mathrm{d} P r_{0}}{2 \mathrm{~d} r_{0}} \lambda^{3}
$$

with further modifications to Eq. (4) as:

$$
\left\{\begin{array}{l}
h=\lambda^{-2} \mathrm{~d} r_{0}=\frac{\mathrm{d} r_{0}}{\lambda^{2}}, \\
r=\lambda r_{0} \Rightarrow \mathrm{d} r=\mathrm{d}\left(\lambda r_{0}\right) .
\end{array}\right.
$$

Since now $\mathrm{d} r$ becomes the deformed thickness $h$, i.e., $h=\mathrm{d} r$, we find that:

$$
\frac{\mathrm{d} r_{0}}{\lambda^{2}}=\mathrm{d}\left(\lambda r_{0}\right)=r_{0} \mathrm{~d} \lambda+\lambda \mathrm{d} r_{0} \Rightarrow \mathrm{d} r_{0}=\frac{r_{0} \mathrm{~d} \lambda}{\lambda^{-2}-\lambda} .
$$

On substituting $\mathrm{d} r_{0}$ from Eq. (15) into (13) we obtain:

$$
\mathrm{d} P=\sigma \frac{2 \lambda^{-4}}{\lambda^{-3}-1} \mathrm{~d} \lambda
$$

By using equation (5) for $\sigma$, Eq. (16) may be re-written to give an explicit relationship for the inflation pressure $P$ and inflation stretch $\lambda$ as:

$$
\mathrm{d} P=4 \frac{\lambda^{-2}-\lambda^{-8}}{\lambda^{-3}-1} \frac{\partial W}{\partial I_{1}} \mathrm{~d} \lambda .
$$

Using Eq. (7) and noting that $I_{1}=2 \lambda^{2}+\lambda^{-4}$, the final form of the inflation pressure - stretch relationship in our model for thick spherical shells may be arrived at as:

$$
\mathrm{d} P=-\frac{2}{3} \mu\left(\lambda^{-2}+\lambda^{-5}\right)\left(\frac{2 \lambda^{2}+\lambda^{-4}-9 N}{2 \lambda^{2}+\lambda^{-4}-3 N}\right) \mathrm{d} \lambda .
$$

Note that the inflation pressure $P$ is obtained by (numerically) integrating Eq. (18) from $P=0$ at $\lambda=1$ to $-P$ in the inner surface.

From the relationship in Eq. (18) we find that Eq. (10) $)_{1}$ has only one real root for all $N>$ 1 in $\lambda>1$, and therefore our model does not predict a limit-point/inflation-jump behaviour for the inflation of thick-walled spherical shells. Note that the solution to Eq. $(10)_{1}$ at any given $N$ renders the maximum stretch $\lambda$ at which the limiting chain extensibility will be reached. Therefore, always a monotonic and stable inflation is predicted for thick-walled spherical shells. Normalised inflation pressure curves produced by Eq. (18) using various values of $N$ are presented in Fig. 3, reinforcing the prediction of a stable and monotonic inflation for all valid ranges of $N$ and deformation.

\subsection{Pressure-Inflation Relationship for Thin Cylindrical Tube Shells}

We now consider the inflation of long closed-end thin cylindrical tubes using a similar approach used in $\S \$ 2.1$ and 2.2. However, unlike the problem of spherical balloons, two principal stretches appear in the analysis of cylindrical shells, namely the circumferential stretch $\lambda_{\theta}$ and the axial stretch $\lambda_{z}$. See Fig. 4 for the definition of cylindrical coordinates and directions. 


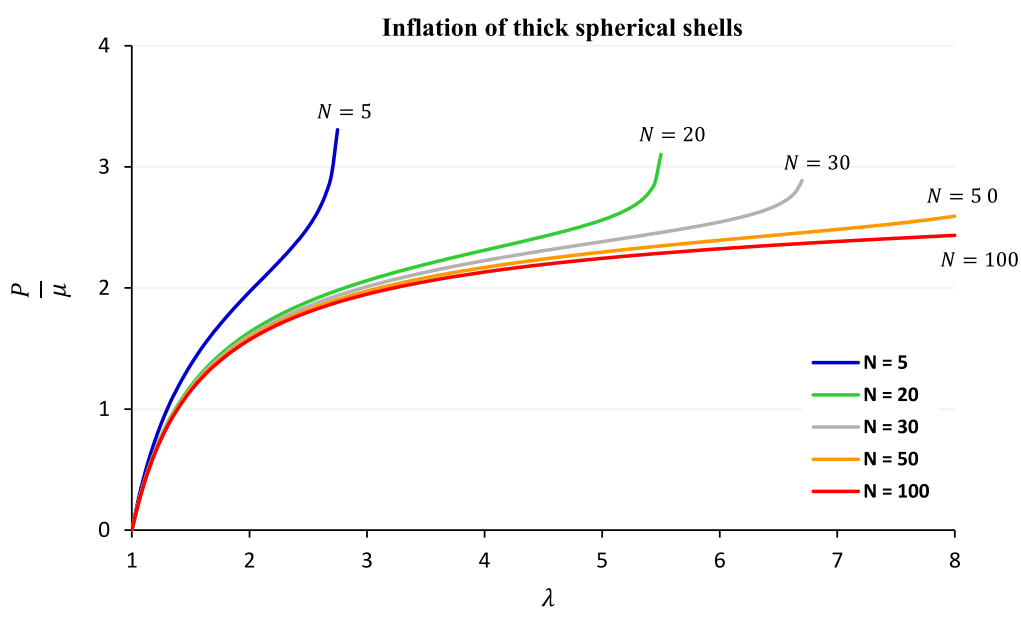

Fig. 3 Normalised inflation pressure versus stretch for a thick spherical balloon shell using our proposed model in Eq. (18) at various values of $N$. Note that for all valid values of $N>1$, the model always predicts a monotonic and stable inflation

Fig. 4 Cylindrical coordinates and directions as used in our derivations

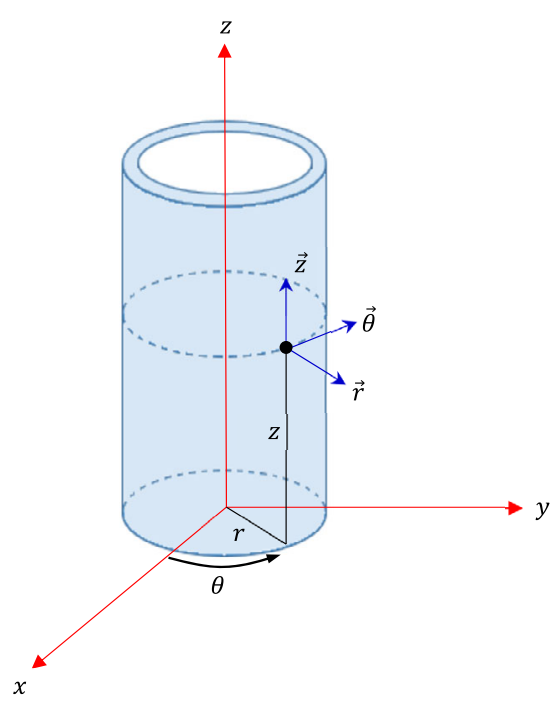

The counterpart to Eq. (3) for cylinders is (see [27] for a detailed analysis):

$$
\left\{\begin{array}{l}
P=\frac{2 h}{r} \sigma_{z}, \\
P=\frac{h}{r} \sigma_{\theta},
\end{array}\right.
$$

using the axial and hoop stresses, respectively. Subject to incompressibility we have $\lambda_{z} \lambda_{\theta} \lambda_{r}=1$ and Eq. (4) is accordingly adjusted to: 


$$
\left\{\begin{array}{l}
\frac{r}{r_{0}}=\lambda_{\theta}, \\
\frac{h}{h_{0}}=\lambda_{\theta}^{-1} \lambda_{z}^{-1},
\end{array}\right.
$$

and $I_{1}=\lambda_{\theta}^{2}+\lambda_{z}^{2}+\lambda_{\theta}^{-2} \lambda_{z}^{-2}$. From Eq. (5) we also get:

$$
\left\{\begin{array}{l}
\sigma_{\theta}=2 \frac{\partial W}{\partial I_{1}}\left(\lambda_{\theta}^{2}-\lambda_{\theta}^{-2} \lambda_{z}^{-2}\right), \\
\sigma_{z}=2 \frac{\partial W}{\partial I_{1}}\left(\lambda_{z}^{2}-\lambda_{\theta}^{-2} \lambda_{z}^{-2}\right) .
\end{array}\right.
$$

On substituting Eqs. (20) and (21) into (19) the final form of the inflation pressure - stretch relationship in our model for thin cylindrical shells may be arrived at as:

$$
\left\{\begin{array}{l}
P=\frac{h_{0}}{3 r_{0}} \mu\left(\lambda_{z}^{-1}-\lambda_{\theta}^{-4} \lambda_{z}^{-3}\right)\left(\frac{\lambda_{\theta}^{2}+\lambda_{z}^{2}+\lambda_{\theta}^{-2} \lambda_{z}^{-2}-9 N}{\lambda_{\theta}^{2}+\lambda_{z}^{2}+\lambda_{\theta}^{-2} \lambda_{z}^{-2}-3 N}\right) \\
P=\frac{2 h_{0}}{3 r_{0}} \mu\left(\lambda_{z} \lambda_{\theta}^{-2}-\lambda_{\theta}^{-4} \lambda_{z}^{-3}\right)\left(\frac{\lambda_{\theta}^{2}+\lambda_{z}^{2}+\lambda_{\theta}^{-2} \lambda_{z}^{-2}-9 N}{\lambda_{\theta}^{2}+\lambda_{z}^{2}+\lambda_{\theta}^{-2} \lambda_{z}^{-2}-3 N}\right) .
\end{array}\right.
$$

Note that the left hand-side of the Eqs. $(22)_{1}$ and $(22)_{2}$, i.e., the inflation pressure $P$, are the same, which renders an interesting relationship between $\lambda_{\theta}$ and $\lambda_{z}$ stretches as also noted in [23]:

$$
2 \lambda_{z}^{2}-\lambda_{\theta}^{2}-\lambda_{\theta}^{-2} \lambda_{z}^{-2}=0
$$

with only the following valid roots over the range of deformation:

$$
\left\{\begin{array}{l}
\lambda_{\theta}=\sqrt{\frac{\sqrt{\lambda_{z}^{6}-1}}{\lambda_{z}}+\lambda_{z}^{2}} \\
\lambda_{z}=\frac{1}{2} \sqrt{\frac{\sqrt{\lambda_{\theta}^{6}+8}}{\lambda_{\theta}}+\lambda_{\theta}^{2}}
\end{array}\right.
$$

It may, however, be more convenient to use the auxiliary volume variable $v$ as we did in $\S 2.1$. In this case, the volume expansion ratio $v$ will be $v=\lambda_{\theta}^{2} \lambda_{z}$. Using this new inflation measure, Eq. (22) may be simply re-written as:

$$
P=\frac{\sqrt[3]{2}}{3} \frac{h_{0}}{r_{0}} \mu \frac{v^{2}-1}{v \sqrt[3]{v^{2}\left(v^{2}+1\right)}} \frac{I_{1}-9 N}{I_{1}-3 N},
$$

with $I_{1}=3\left(\frac{v^{2}+1}{2 v}\right)^{\frac{2}{3}}$.

It is now possible to calculate the critical value of the model parameter $N$ where the limitpoint instability won't be exhibited in the inflation of thin spherical shells, using Eqs. (10) and (25). It can be shown that the conditions in Eq. (10) are held if:

$$
2 v^{12}-192 N v^{10}+\left(864 N^{2}+192 N-22\right) v^{8}-\left(3456 N^{2}-960 N+48\right) v^{6}
$$




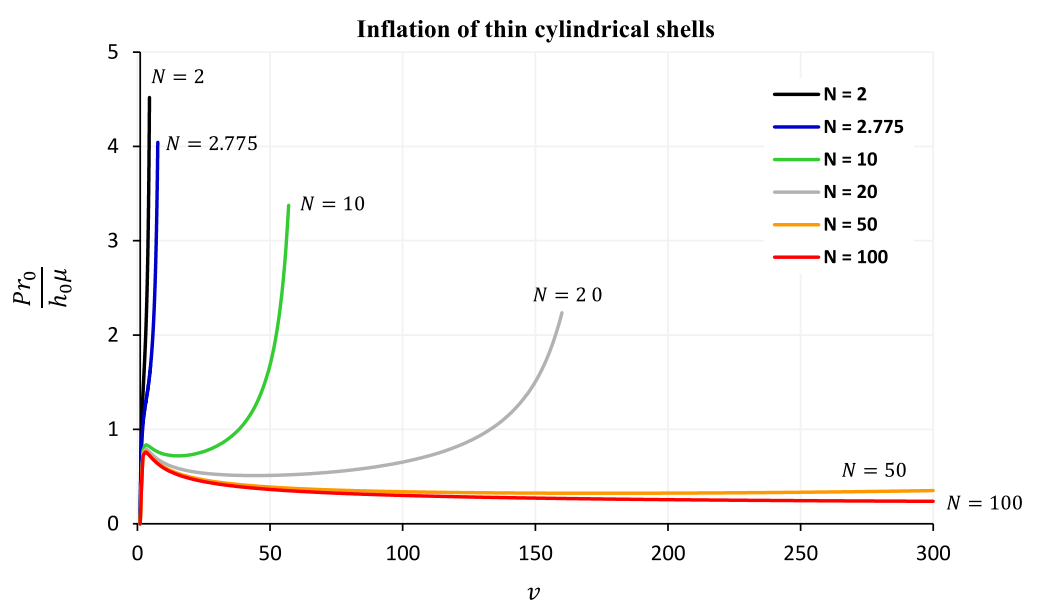

Fig. 5 Normalised inflation pressure versus volume for a thin cylindrical tube shell using our proposed model in Eq. (25) at various values of $N$. For $N<2.775$, it is clear that the inflation is monotonic and stable; while for $N>2.775$ both limit-point and inflation-jump instabilities are evident

$$
-\left(864 N^{2}-576 N+42\right) v^{4}-16 v^{2}-2=0,
$$

and we find that Eq. (26) has two real roots in $\lambda>1$ for $N$ above the threshold value $N_{t}$ as:

$$
N>N_{t} \cong 2.775
$$

For $N<2.775$, Eq. (25) and by extension Eq. (22) predicts a monotonic and stable inflation of thin cylindrical shells. For $N>2.775$, the inflation pressure curves clearly exhibit a maximum pressure, i.e., the limit-point instability, and the second ascending branch, i.e., the inflation-jump instability. Normalised inflation pressure curves produced by Eq. (25) using various values of $N$ below and above $N_{t}$ are presented in Fig. 5, reinforcing the existence of the threshold $N_{t}$ value.

Remark 1 From the relatively low values of $N_{t}$ in Eqs. (12) and (27) it may be inferred that for rubber-like materials with sufficiently low extensibility, stable inflation is predicted for both spherical and cylindrical thin shells. This result is consistent with that of [23] in which the Gent model has also been shown to report a relatively low threshold value of $J_{m}$, where $J_{m}$ is the extensibility limiting factor. A similar trend may also be extended to biological soft tissues. Our early observations regarding the values of $N$ when modelling soft tissues are that they are typically low (see, e.g., [4] for tendons with $2.05<N<3.70$ ). The literature also reports on low values of $J_{m}$ in human arterial tissues, e.g., [21], with $0.4<J_{m}<2.3$. One may consider the relationship between $J_{m}$ and $N$ to be $J_{m}=3(N-1)$, and therefore the value of $N$ for arteries will fall in the range of $1.13<N<1.77$. Separately, some studies have shown no instability in the inflation of human arteries (see, e.g., [18]). Therefore, the reported stable inflation behaviour for those arteries are consistent with the threshold value $N_{t}$ for cylindrical thin shells predicted by our model. However, if future studies reveal that arterial tissues may report higher values of $N$ but still exhibit a stable inflation, then perhaps the assumption of thick-walled cylindrical tubes maybe a more appropriate assumption see $\$ 2.4$. 


\subsection{Pressure-Inflation Relationship for Thick Cylindrical Tube Shells}

In a similar manner to $\$ 2.2$, here we derive and present the inflation pressure - deformation relationships for thick cylindrical shells. Accordingly, the internal pressure $P$ required to inflate a thick-walled cylindrical shell may be obtained by integrating the contribution of $\mathrm{d} P$ from concentric thin-walled cylindrical shells with wall thickness $\mathrm{d} r_{0}$. Relationships in Eq. (20) may be re-written as:

$$
\left\{\begin{array}{l}
\frac{r}{r_{0}}=\lambda_{\theta} \Rightarrow r=r_{0} \lambda_{\theta} \Rightarrow \mathrm{d} r=\mathrm{d}\left(r_{0} \lambda_{\theta}\right) \\
h=\frac{\mathrm{d} r_{0}}{\lambda_{\theta} \lambda_{z}}
\end{array}\right.
$$

Again, since $\mathrm{d} r$ becomes the deformed thickness $h$, i.e., $h=\mathrm{d} r$, we find that:

$$
\frac{\mathrm{d} r_{0}}{\lambda_{\theta} \lambda_{z}}=\mathrm{d}\left(\lambda r_{0}\right)=r_{0} \mathrm{~d} \lambda_{\theta}+\lambda_{\theta} \mathrm{d} r_{0} \Rightarrow \mathrm{d} r_{0}=\frac{r_{0} \mathrm{~d} \lambda_{\theta}}{\lambda_{\theta}^{-1} \lambda_{z}^{-1}-\lambda_{\theta}} .
$$

Similarly, the inflation pressure - Cauchy stress relationships in Eq. (19) may be accordingly re-written as:

$$
\left\{\begin{array}{l}
\mathrm{d} P=\frac{2 \lambda_{\theta}^{-1} \lambda_{z}^{-1} \mathrm{~d} r_{0}}{\lambda_{\theta} r_{0}} \sigma_{z}, \\
\mathrm{~d} P=\frac{\lambda_{\theta}^{-1} \lambda_{z}^{-1} \mathrm{~d} r_{0}}{\lambda_{\theta} r_{0}} \sigma_{\theta} .
\end{array}\right.
$$

By substituting for $\mathrm{d} r_{0}$ from Eq. (29) into (30) and on using Eq. (21), we arrive at the differential form of the inflation pressure - stretch relationships in our model for thickwalled cylindrical shells as:

$$
\left\{\begin{array}{l}
\mathrm{d} P=-\frac{2 \mu\left(\lambda_{\theta}^{2} \lambda_{z}^{4}-1\right)}{3 \lambda_{\theta}^{3} \lambda_{z}^{2}\left(\lambda_{\theta}^{2} \lambda_{z}-1\right)}\left(\frac{\lambda_{\theta}^{2}+\lambda_{z}^{2}+\lambda_{\theta}^{-2} \lambda_{z}^{-2}-9 N}{\lambda_{\theta}^{2}+\lambda_{z}^{2}+\lambda_{\theta}^{-2} \lambda_{z}^{-2}-3 N}\right) \mathrm{d} \lambda_{\theta}, \\
\mathrm{d} P=-\frac{\mu\left(\lambda_{\theta}^{2} \lambda_{z}+1\right)}{3 \lambda_{\theta}^{3} \lambda_{z}^{2}}\left(\frac{\lambda_{\theta}^{2}+\lambda_{z}^{2}+\lambda_{\theta}^{-2} \lambda_{z}^{-2}-9 N}{\lambda_{\theta}^{2}+\lambda_{z}^{2}+\lambda_{\theta}^{-2} \lambda_{z}^{-2}-3 N}\right) \mathrm{d} \lambda_{\theta} .
\end{array}\right.
$$

Note that the inflation pressure $P$ is obtained by (numerically) integrating the relationships in Eq. (31) from $P=0$ at $\lambda_{\theta}=1$ to $-P$ in the inner surface.

Similar to the case of thin cylindrical shells, it may be more convenient to use the volume expansion measure $v$ instead of the principal stretches, and thus Eq. (31) may be simplified to the following inflation pressure - volume relationship:

$$
\mathrm{d} P=-\frac{\sqrt[3]{2} \mu(v+1)\left(v^{2}+2\right)}{9 v\left(v^{2}+1\right) \sqrt[3]{v^{2}\left(v^{2}+1\right)}} \frac{I_{1}-9 N}{I_{1}-3 N} \mathrm{~d} v,
$$

with $I_{1}=3\left(\frac{v^{2}+1}{2 v}\right)^{\frac{2}{3}}$.

From the relationship in Eq. (32) we find that Eq. (10) $)_{1}$ has only one real root for all $N>$ 1 in $\lambda>1$, and therefore our model predicts a monotonic and stable inflation for thick-walled cylindrical shells. Normalised inflation pressure curves produced by Eq. (32) using various values of $N$ are presented in Fig. 6, reinforcing the prediction of a stable and monotonic inflation for all valid ranges of $N$ and deformation. 


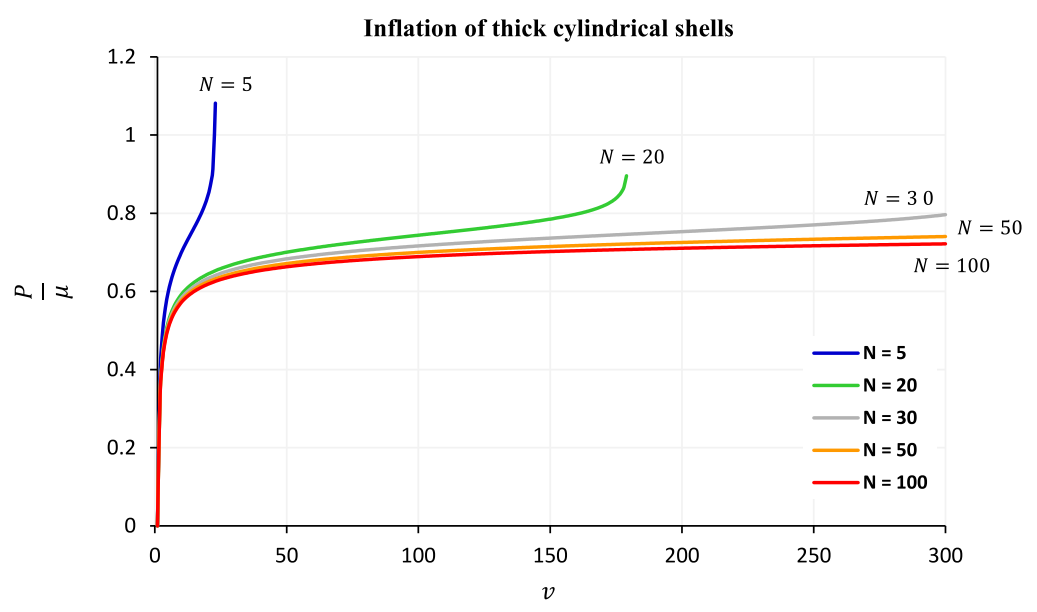

Fig. 6 Normalised inflation pressure versus volume expansion ratio for thick cylindrical tube shells using our proposed model in Eq. (32) at various values of $N$. Note that for all valid values of $N>1$, the model always predicts a monotonic and stable inflation

\section{Application to Experimental Data}

In this section we apply the inflation equations derived in $\$ 2$ to extant experimental datasets for modelling the inflation phenomena observed in rubbers. The scope of the experimental data entails the inflation of spherical balloons and cylindrical tubes, procured and collated from studies across a span of three centuries, from Mallock's early work in 1891 [26] to that of Holzapfel [18] and Mangan and Destrade [27] in the 21st century! The tabulated collated pressure - inflation data from each study are presented in Appendix.

To find the best model fit to each considered set of data, the designated relevant model equations were curve fitted to the data by minimising the residual sum of squares (RSS) function defined as: $\mathrm{RSS}=\sum_{i}\left(P^{\text {model }}-P^{\text {experiment }}\right)_{i}^{2}$, where $i$ is the number of data points. This minimisation was achieved via an in-house developed code in MATLAB ${ }^{\circledR}$, using the genetic algorithm (GA) toolbox. The values of $\mathrm{R}^{2}$ for each fit were calculated first by establishing the Coefficient of Correlation using a built-in MATLAB function and then squaring that value.

\subsection{Spherical Balloon Shells}

Through [14] we first became aware of Mallock's early work in studying the inflation instabilities in what has been referred to as India rubber [26]. Therein he reports inflation pressure $(P)$ - volume ratio $(v)$ data for a spherical thin shell, with $\frac{h_{0}}{r_{0}}=0.0125$. Modelling results for this dataset using Eq. (9) are shown in Fig. 7. The model parameters are $\mu=390$ $\mathrm{kPa}$ and $N=7.28 \times 10^{14}$, with $\mathrm{R}^{2}=0.96$.

Mallock's data only includes the onset of limit-point instability, and hence using the model we were able to predict the critical (maximum) pressure and the behaviour beyond this point (see the right panel in Fig. 7). The maximum pressure reported in the data is 5.70 $\mathrm{kPa}$, while that of the model corresponds to $5.79 \mathrm{kPa}$, showing a favourable agreement. Note that model parameters for all considered datasets in this section are summarised in Table 1.

The second dataset we consider here is due to Beatty (1987) [5], where the inflation pressure $(P)-\operatorname{stretch}(\lambda)$ data for a "typical balloon inflation experiment" has been reported. 


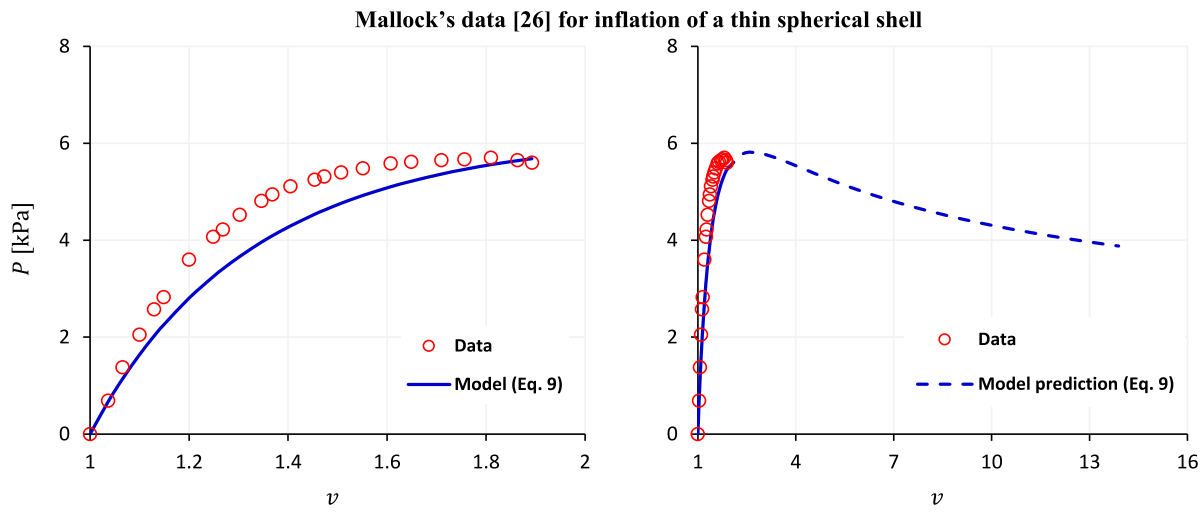

Fig. 7 Modelling results for the inflation of the India rubber spherical balloon shell using Mallock (1891) data [26] and Eq. (9). The left panel shows the direct fitting result and the right panel shows the model prediction of the critical pressure and beyond

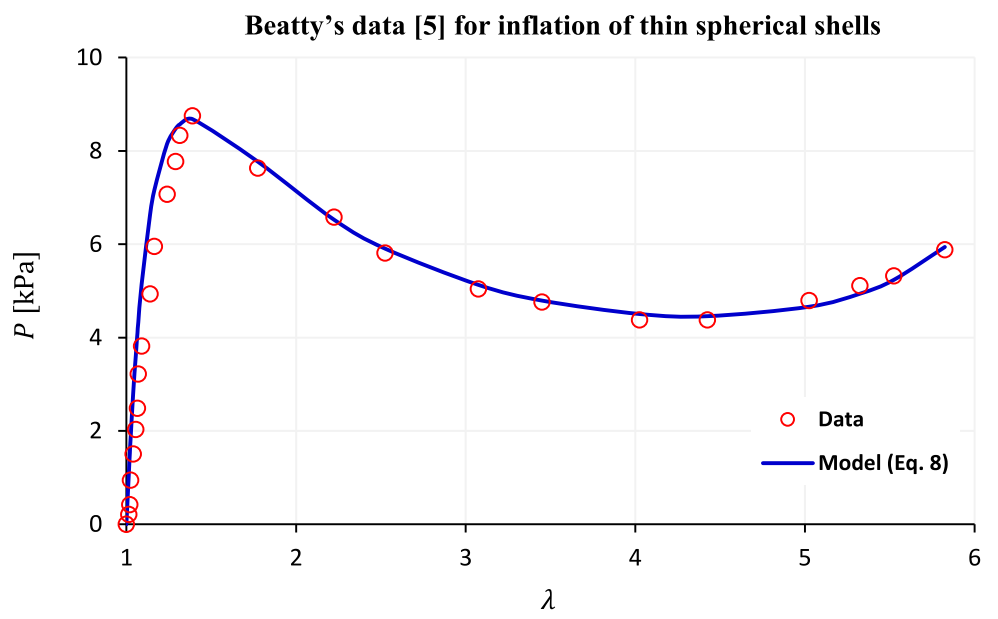

Fig. 8 Modelling result for a "typical balloon inflation experiment" reported in [5] using Eq. (8)

That study does not present the value of $\frac{h_{0}}{r_{0}}$ ratio, however we assume a middle point between that of [26] and [27] as 0.02. Using Eq. (8), the modelling result for this dataset is presented in Fig. 8, reporting a favourable agreement between the experimental data and the model. The modelling parameters are $\mu=340 \mathrm{kPa}$ and $N=32.38$, with $\mathrm{R}^{2}=0.98$ (see Table 1). The reported experimental maximum pressure is $8.75 \mathrm{kPa}$, versus $8.68 \mathrm{kPa}$ of the model, showing a good agreement.

The third dataset we present here is for the inflation of vulcanized natural rubber spherical balloon specimens due to Rivlin and Saunders (1951) [34]. Therein they report on the normalised inflation pressure values in terms of $\frac{P r}{4 h_{0}\left(1-\lambda^{-6}\right)}$. Since our formulation of inflation pressure in Eq. (8) is more readily amenable to $\frac{3 P r_{0}}{2 h_{0}}$ normalisation, we undertook to convert the values in [34] by a factor of $6\left(\lambda^{-1}-\lambda^{-7}\right)$; see Appendix for the converted values. The modelling results are shown in Fig. 9 , with $\mu=0.39 \mathrm{MPa}, N=9.78 \times 10^{14}$ and $R^{2}=0.97$ 


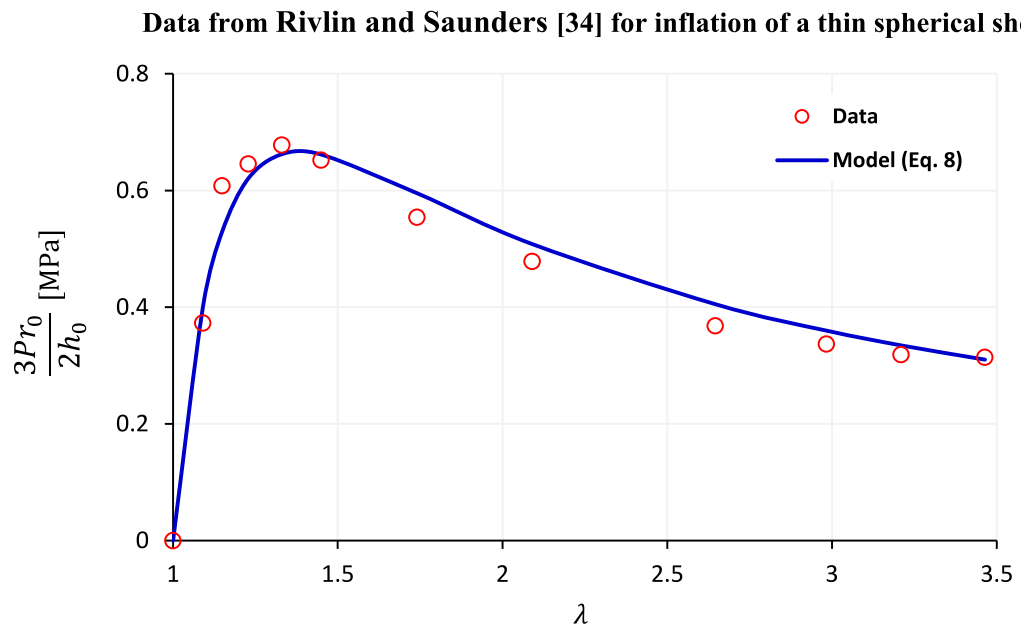

Fig. 9 Modelling result for vulcanized natural rubber spherical balloon specimens due to Rivlin and Saunders (1951) [34] using Eq. (8). Note the adjustment required for normalisation of the pressure values to $\frac{3 P r_{0}}{2 h_{0}}$

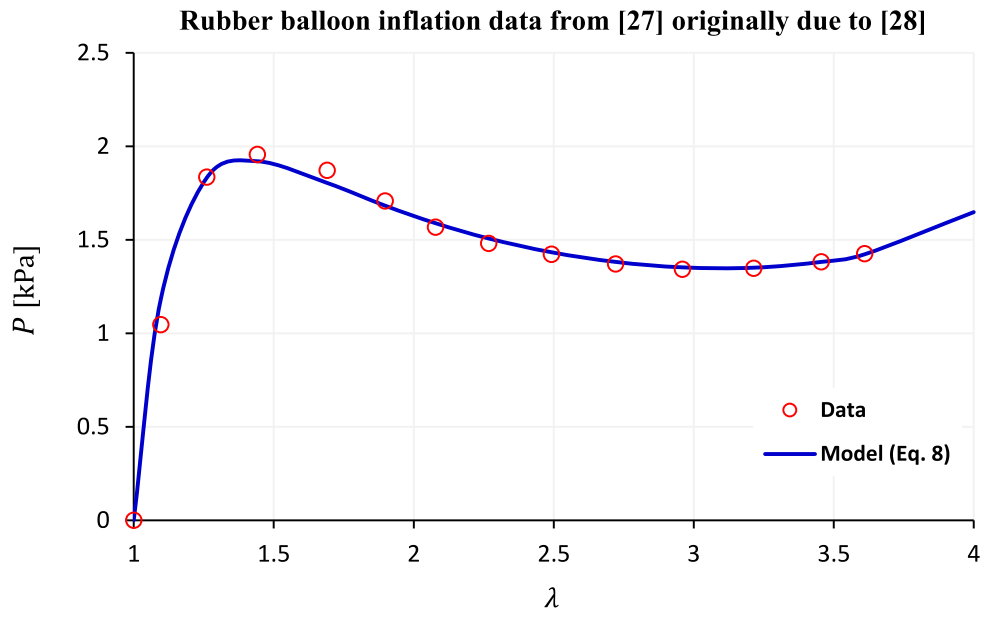

Fig. 10 Modelling result for inflation of typical rubber balloons of "the dime-store variety" using Eq. (8). Data from [27] but originally due to Merritt and Weinhaus (1978) [28]

(see Table 1). The experimental maximum (pressure) data reports a value of $0.68 \mathrm{MPa}$ and that of the model is $0.66 \mathrm{MPa}$, showing a close proximity.

Next we consider the inflation data originally due to Merritt and Weinhaus (1978) [28], reported in [27], for rubber balloons of "the dime-store variety". The data was kindly provided to us by Professor Destrade, where an average $\frac{h_{0}}{r_{0}}$ ratio of 0.026 was considered [27]. Using Eq. (8), the modelling result for this dataset is presented in Fig. 10, where an excellent agreement between the data and the model predictions are observed. The modelling parameters are $\mu=56.10 \mathrm{kPa}$ and $N=16.33$, with $\mathrm{R}^{2}=0.99$ (see Table 1). The reported 


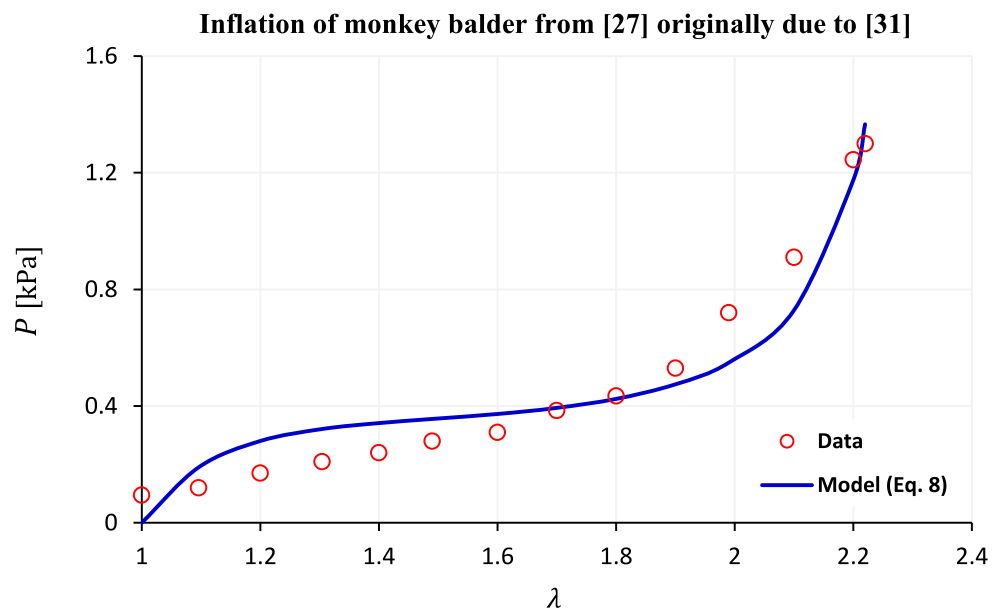

Fig. 11 Modelling result for the inflation of the monkey bladder using Eq. (8) and the data in [27], originally due to Osborne 1909 [31]

Table 1 Model parameter values for the inflation of spherical balloon shells data

\begin{tabular}{llll}
\hline & $N[-]$ & $\mu[\mathrm{kPa}]$ & $\mathrm{R}^{2}$ \\
\hline Mallock (1891) data [26] & $7.28 \times 10^{14}$ & 390 & 0.96 \\
Beatty (1987) data [5] & 32.38 & 340 & 0.98 \\
Rivlin and Saunders (1951) data [34] & $9.78 \times 10^{14}$ & 360 & 0.97 \\
Merritt and Weinhaus (1978) data [28] & 16.33 & 56.20 & 0.99 \\
Osborne (1909) data [31] & 3.62 & 1.30 & 0.94 \\
\hline
\end{tabular}

experimental maximum pressure is $1.96 \mathrm{kPa}$, versus $1.92 \mathrm{kPa}$ of the model, showing a very good agreement.

Finally, to include a flavour of soft tissue biomechanics, we also consider in this section the application of our opposed model to inflation of hollow viscera, and in particular the monkey bladder as originally carried out by Osborne 1909 [31]. This dataset was considered in [27] and here we use that data. While the original $\frac{h_{0}}{r_{0}}$ ratio for the specimens has not been reported in [31], Mangan and Destrade stipulated a ratio of $\sim 0.15$ [27]. Using this value and Eq. (8), the experimental data versus modelling results are presented in Fig. 11. Model parameters are $\mu=1.30 \mathrm{kPa}$ and $N=3.62$, with $\mathrm{R}^{2}=0.94$ (see Table 1). Since the value of $N$ is below that of $N_{t} \cong 5.603$ given in Eq. (12) as the threshold value for exhibiting the limit-point instability in the inflation of thin spherical shells, a stable inflation has been predicted by the model. As noted in [27], Osborne's data does not originally start from zero pressure, and potential anisotropic effects are not accounted for in the current inflation pressure formulations. Considering these two limitations, the model is able to provide a reasonable characterisation of the data. Shortcomings of other models such as that of Mooney and Gent to describe this particular dataset have been discussed and presented in [27].

Table 1 summarises all the values of the model parameters obtained for all the fits presented in this section. 
Mallock's data [26] for inflation of thin cylindrical shells
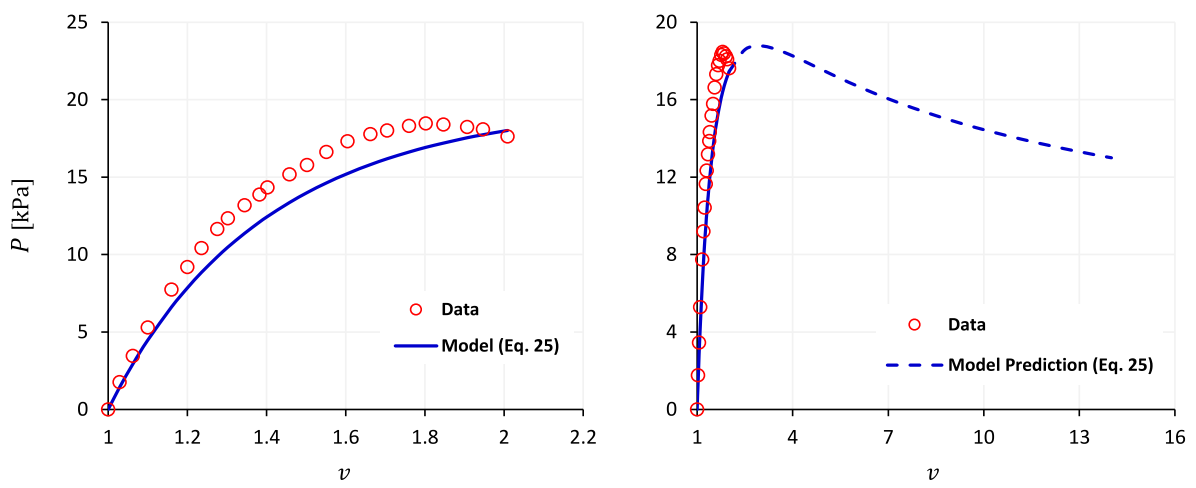

Fig. 12 Modelling results for the inflation of the India rubber cylindrical tube shell using Mallock (1891) data [26] and Eq. (25). The left panel shows the direct fitting result and the right panel shows the model prediction of the critical pressure and beyond

\subsection{Cylindrical Tube Shells}

Similar to the previous section, we start by presenting the results for Mallock's data on the inflation of thin India rubber cylindrical shells [26]. The reported $\frac{h_{0}}{r_{0}}$ for these specimens is 0.039 , and the experimental data have been presented as inflation pressure $(P)$ - volume $(v)$. Modelling results for this dataset using Eq. (25) are shown in Fig. 12. The model parameters are $\mu=662 \mathrm{kPa}$ and $N=7.28 \times 10^{14}$, with $\mathrm{R}^{2}=0.97$. As with the spherical balloons, Mallock's data only includes the onset of limit-point instability, and hence using the model we were able to predict the critical (maximum) pressure and the behaviour beyond this point (see the right panel in Fig. 12). The maximum pressure reported in the data is $18.46 \mathrm{kPa}$, while that of the model corresponds to $18.77 \mathrm{kPa}$, showing a close agreement. Note that model parameters for all considered datasets in this section are summarised in Table 2.

The second dataset we consider here is due to Alexander (1971), where experimental data for latex tube inflation have been reported [1]. The considered experimental data set therein have been provided in normalised inflation pressure - circumferential stretch $\left(\lambda_{\theta}\right)$ data, with the normalised pressure being $\frac{P r_{0}}{2 \mu h_{0}}$; see Appendix for the dataset. With this data, we first calculated the axial stretch $\lambda_{z}$ from Eq. (24) 2 and subsequently used Eq. (22), adjusted to account for the normalisation, to model the inflation data. Modelling results are presented in Fig. 13, with model parameter $N=32.05$ and $\mathrm{R}^{2}=0.99$ (see also Table 2).

The dataset from Alexander (1971) [1] is interesting as it only contains data points before the onset of limit-point instability, and then on the second ascending branch, i.e., the inflation-jump instability. Therefore the model has to predict the inflation behaviour between these two instabilities and capture the maximum (normalised) pressure, which is predicted by the model to be at 0.386 . The model appears to provide an excellent agreement with the data.

The third dataset that we analyse here is due to Charrier (1974), where inflation of thin rubber tubes under various axial pre-stretches have been studied [8]. According to the described experimental protocol, an axial force was first applied to the specimen to achieve a pre-set level of axial stretch $\lambda_{z}$. Then the stretch was kept constant while the tube was inflated. Here we consider the inflation data under three fixed axial stretches of $\lambda_{z}=1,1.5$ and 2.5. The pressure data therein has been normalised as $\frac{P r_{0}}{h_{0}}$. The three datasets were simulta- 


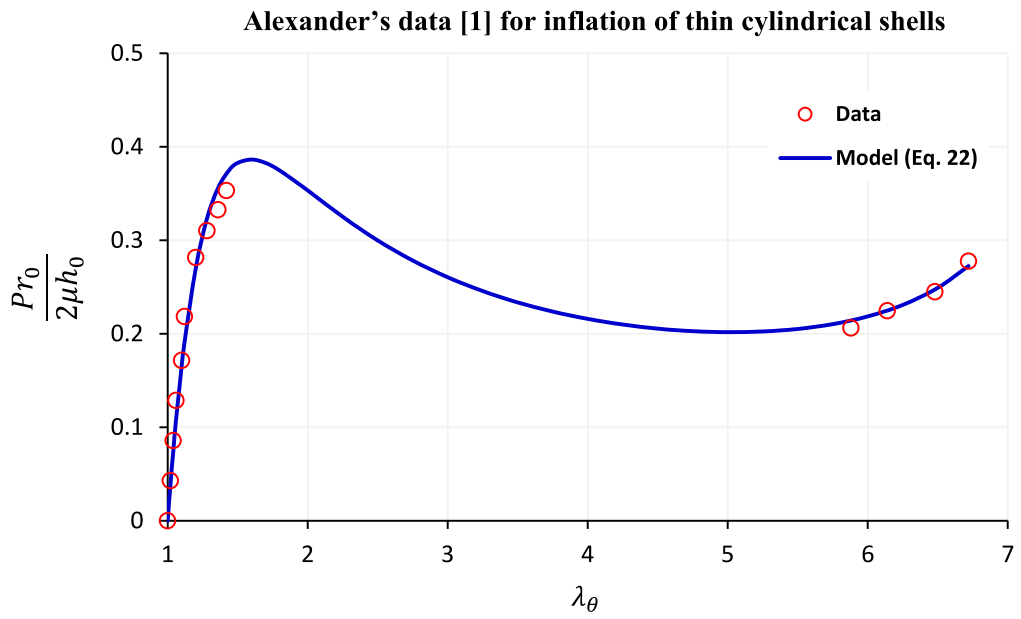

Fig. 13 Modelling result for the inflation of latex tubes due to [1] and using Eq. (22), adjusted to account for the normalisation $\frac{P r_{0}}{2 \mu h_{0}}$

Table 2 Model parameter values for the inflation of cylindrical tube shell data

\begin{tabular}{llllr}
\hline & & $N[-]$ & $\mu[\mathrm{kPa}]$ & $\mathrm{R}^{2}$ \\
\hline Mallock (1891) data [26] & & $7.28 \times 10^{14}$ & 662 & 0.97 \\
Alexander (1971) data [1] & & 32.05 & - & 0.99 \\
Charrier (1974) data [8] & & $5.5 \pm 0.41$ & $690 \pm 30$ & $>0.98$ \\
Holzapfel (2005) data [18] & Rubber & 27.44 & 26 & 0.99 \\
& Iliac Artery & 1.0008 & 0.351 & 0.99 \\
Kyriakides and Chang data & 1990 [24] & 37.98 & - & 0.92 \\
& $1991[25]$ & 27.31 & - & 0.99 \\
\hline
\end{tabular}

neously fitted to Eq. (22), adjusted to account for the $\frac{P r_{0}}{h_{0}}$ normalisation, and the modelling results are shown in Fig. 14. The model parameters were established to be $\mu=0.69 \pm 0.03$ $\mathrm{MPa}$ and $N=5.5 \pm 0.41$, with $\mathrm{R}^{2}$ values in excess of 0.98 . The values of model parameters have also been summarised in Table 2 .

Note the interesting feature in this dataset is that due to the application of axial prestretch $\lambda_{z}$, the circumferential stretch $\lambda_{\theta}$ enters the compressive range. This dataset therefore provides a valuable assessment of the model's capability in capturing the inflation process within the compressive range too.

Next we consider data from Holzapfel (2005) on inflation of latex tube specimens with a $\frac{h_{0}}{r_{0}}$ ratio of $\sim 0.36$ [18]. The dataset therein reports on inflation pressure $(P)$ - circumferential stretch $\left(\lambda_{\theta}\right)$. Using Eq. (24) 2 we first calculated the values of $\lambda_{z}$ and subsequently the dataset was modelled using Eq. (22). The modelling result is shown in Fig. 15, with model parameters $\mu=26 \mathrm{kPa}$ and $N=27.49$, reporting an excellent agreement with the data $\left(\mathrm{R}^{2}=0.99\right)$. These values are also summarised in Table 2 . Note that [18] only includes data building up to the onset of the maximum pressure. Using the model it was possible to predict the limit-point and inflation-jump instabilities, as shown (with dotted line) in the right panel of Fig. 15, with the maximum pressure of $7.16 \mathrm{kPa}$. 


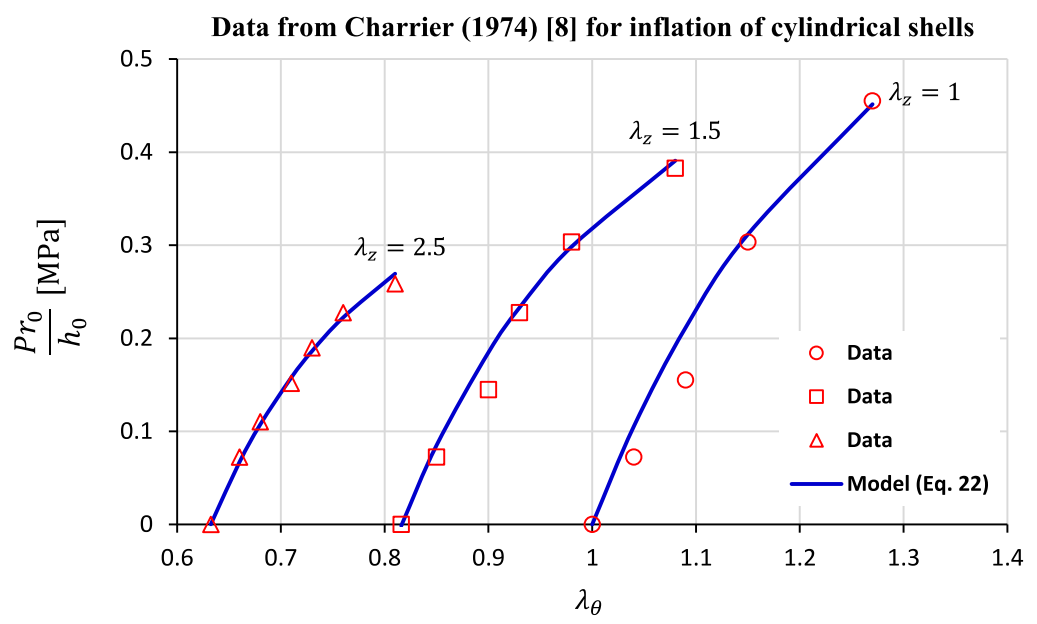

Fig. 14 Modelling results for the inflation of cylindrical rubber tubes under various fixed axial pre-stretch levels: $\lambda_{z}=1,1.5$ and 2.5. Data was collated from [8] and Eq. (22), adjusted to account for the normalisation $\frac{P r_{0}}{h_{0}}$, was used for modelling
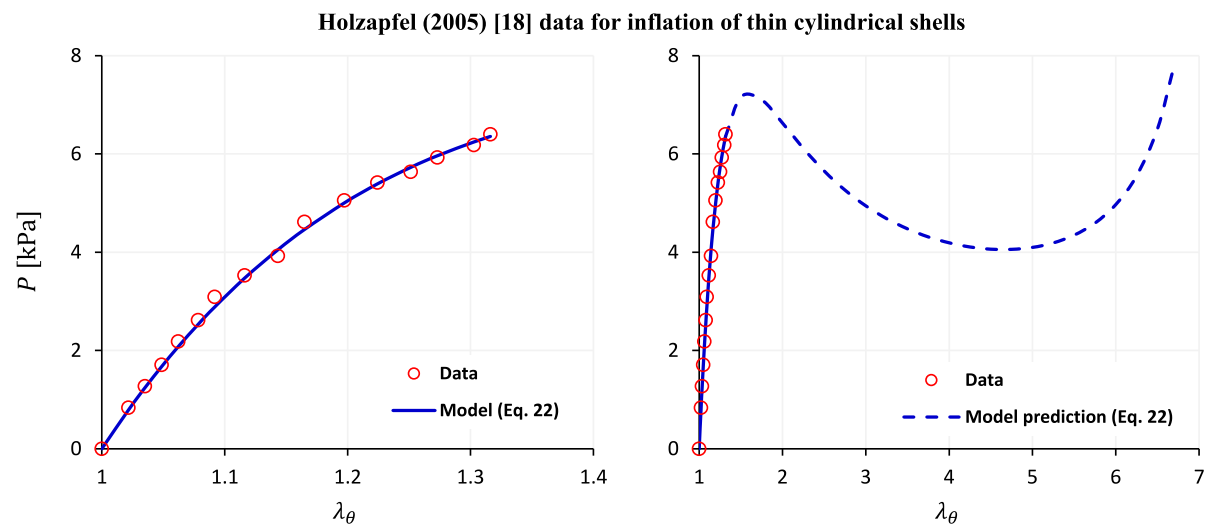

Fig. 15 Modelling results for the inflation of the latex cylindrical tube shell using Holzapfel (2005) data [18] and Eq. (22). The left panel shows the direct fitting result and the right panel shows the model prediction of the critical pressure and beyond

We now consider two datasets from the studies of Kyriakides and Chang [24, 25] on the inflation of commercial natural rubber tubes. In the first study, they report on the normalised inflation pressure $\left(\frac{P R_{0}}{\mu h_{0}}\right)$ - volume ratio $(v)$ data [24]. Note that $R_{0}$ in their representation is the undeformed outside radius of the tube which they report to be $6.35 \mathrm{~mm}$. Given that $r_{0}$ in our formulations is $\left(R_{0}-h_{0}\right)$, the normalisation rendered by our model in Eq. (25) needs to be multiplied by the $\frac{R_{0}}{r_{0}}$ ratio, 1.3368 , to account for the correct normalisation. Accordingly, the data were fitted to Eq. (25), with the modified factor of 1.1368, and the modelling result is shown in Fig. 16(a). As summarised in Table 2, the model parameter $N$ for this dataset is $N=37.98$ with $\mathrm{R}^{2}=0.92$. The experimental maximum (normalised) pressure reports a value of $\sim 0.86$ versus that of the model predicted to be $\sim 0.87$, showing a good agreement. 
Inflation data for cylindrical tubes from [24] and [25]
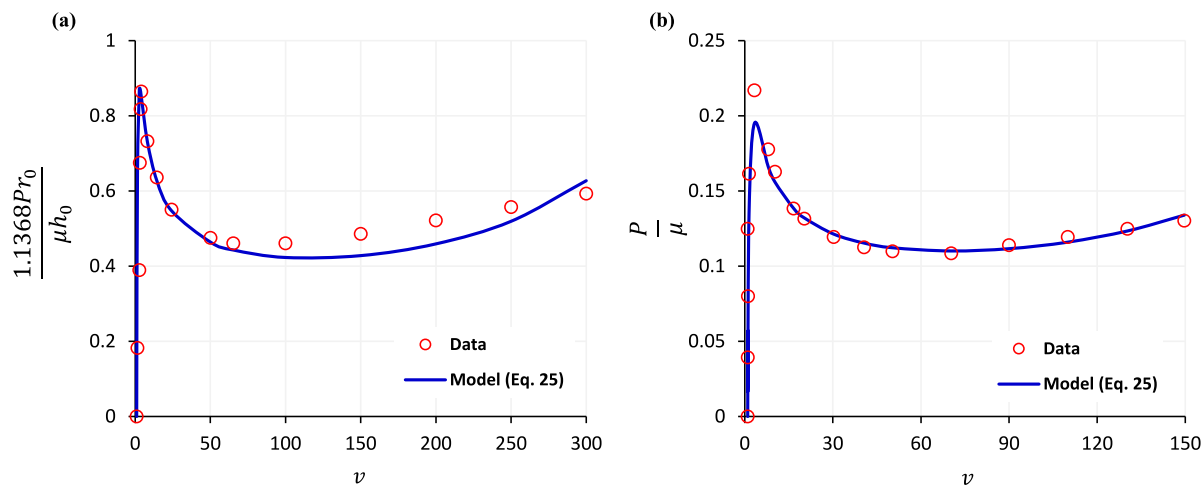

Fig. 16 Modelling results for the inflation of commercial natural rubber tubes using Eq. (25): (a) data from [24]; and (b) from [25]

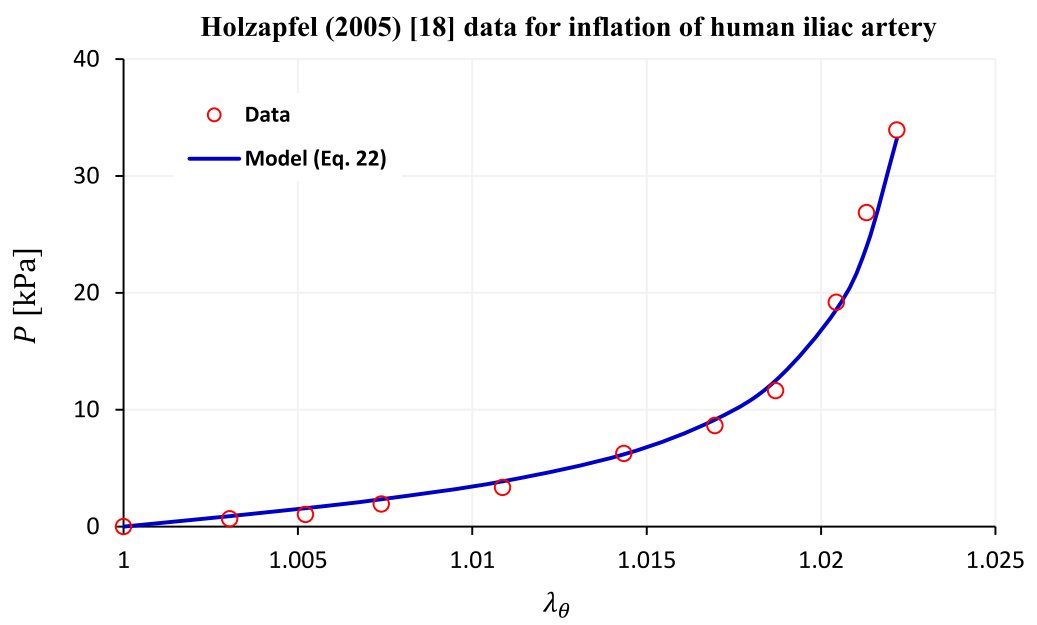

Fig. 17 Modelling result for inflation of human iliac artery from Holzapfel (2005) data [18] using Eq. (22)

The second of the studies, i.e., [25], conducts inflation experiments on similar tubes and reports the normalised pressure $\left(\frac{P}{\mu}\right)$ - volume ratio $(v)$ data. Using Eq. (25), subject to rearrangements required to produce the normalisation, the modelling result is shown in Fig. 16(b), with model parameter $N=27.31$ and $\mathrm{R}^{2}=0.99$ (see Table 2). While the data is generally captured well, note that the experimental maximum (normalised) pressure value is $\sim 0.22$ while that of the model prediction is $\sim 0.19$.

Finally, similar to $\$ 3.1$, we close this section with a soft tissue biomechanics application. Holzapfel (2005) reports on the inflation of human iliac arterial samples [18], where the inflation pressure $(P)$ - circumferential stretch $\left(\lambda_{\theta}\right)$ data have been presented for specimens with a $\frac{h_{0}}{r_{0}}$ ratio of 0.25 . Using Eq. $(24)_{2}$ we calculated the values of $\lambda_{z}$ and then via Eq. (22) modelled the inflation data. The modelling result is shown in Fig. 17, with parameter values of $\mu=0.351 \mathrm{kPa}$ and $N=1.0008$, showing an excellent agreement $\left(\mathrm{R}^{2}=0.99\right)$. Model parameters for this fitting have also been summarised in Table 2. While arterial tissues are 
anisotropic which clearly is not truly captured by the current form of our proposed model, note that the value of $N$ is below the threshold value $N_{t} \cong 2.775$ given in Eq. (27), and therefore the model correctly predicts a stable and monotonic inflation in accordance with experimental observations.

Table 2 summarises all the values of the model parameters obtained for all the fits presented in this section.

\subsection{A Comparison with the Gent Model}

As underlined in [3], the functional form of our proposed $W$ in Eq. (1) is similar to that of the Gent [12]:

$$
W_{\mathrm{G}}=-\frac{\mu_{0}}{2} J_{m} \ln \left(1-\frac{I_{1}-3}{J_{m}}\right),
$$

where $\mu_{0}$ is the infinitesimal shear modulus and $J_{m}$ is a model parameter indicative of the chain extensibility limit. Upon using Eqs. (6) and (21), the ensuing pressure - inflation relationships for (thin) spherical and cylindrical Gent shells may be derived explicitly as:

$$
P=-2 \frac{h_{0}}{r_{0}} \mu_{0}\left(\lambda^{-1}-\lambda^{-7}\right)\left(\frac{J_{m}}{2 \lambda^{2}+\lambda^{-4}-3-J_{m}}\right),
$$

for thin spherical and

$$
P=-\frac{2 h_{0}}{r_{0}} \mu_{0}\left(\lambda_{z}^{-1}-\lambda_{\theta}^{-4} \lambda_{z}^{-3}\right)\left(\frac{J_{m}}{2 \lambda^{2}+\lambda^{-4}-3-J_{m}}\right),
$$

for thin cylindrical shells in inflation.

Since the Gent model also predicts the limit-point and inflation-jump instabilities, and is well-established in the literature, a question may arise as to what advantages our proposed model may offer over the Gent model. To address this point, in addition to reminding the reader that our proposed model has a direct structural root and is not purely phenomenological as the Gent model, we undertook to fit both models to the datasets considered in this study. For brevity, we refrain to replicate all the modelling results and present here by way of example the comparison of both models in capturing the spherical balloon inflation data due to Beatty [5] and Mangan and Destrade [27], as well as that of a cylindrical tube from Alexander [1].

Accordingly, Eqs. (8) and (34) for spherical balloon inflation, and their counterpart pairs in Eqs. (22) 1 and (35) for cylindrical tubes, were fitted to the data by the similar manner described in $\S 3$, subject to the required adjustments to match the data normalisation. The results are shown in Fig. 18. Continuous lines represent our model's predictions, while the dashed lines are those of Gent's.

These sample comparisons make an interesting reading: while may be esoteric, our proposed model provides a more accurate prediction of the experimental data compared with that of the Gent model. This observation may be further reinforced when comparing the goodness of fit parameters such as the $\mathrm{R}^{2}$ and RSS values between the two model fittings. Starting with Beatty's data [5], both models produce $\mathrm{R}^{2}=0.98$; however the RSS values are 0.018 for our model versus 0.020 of the Gent. For the spherical balloon inflation data due to [27], the $\mathrm{R}^{2}$ value of the fit provided by our model is 0.99 , while that of the Gent is 0.98 , with RSS values being 0.027 and 0.029 , respectively. Similarly, for the inflation of 

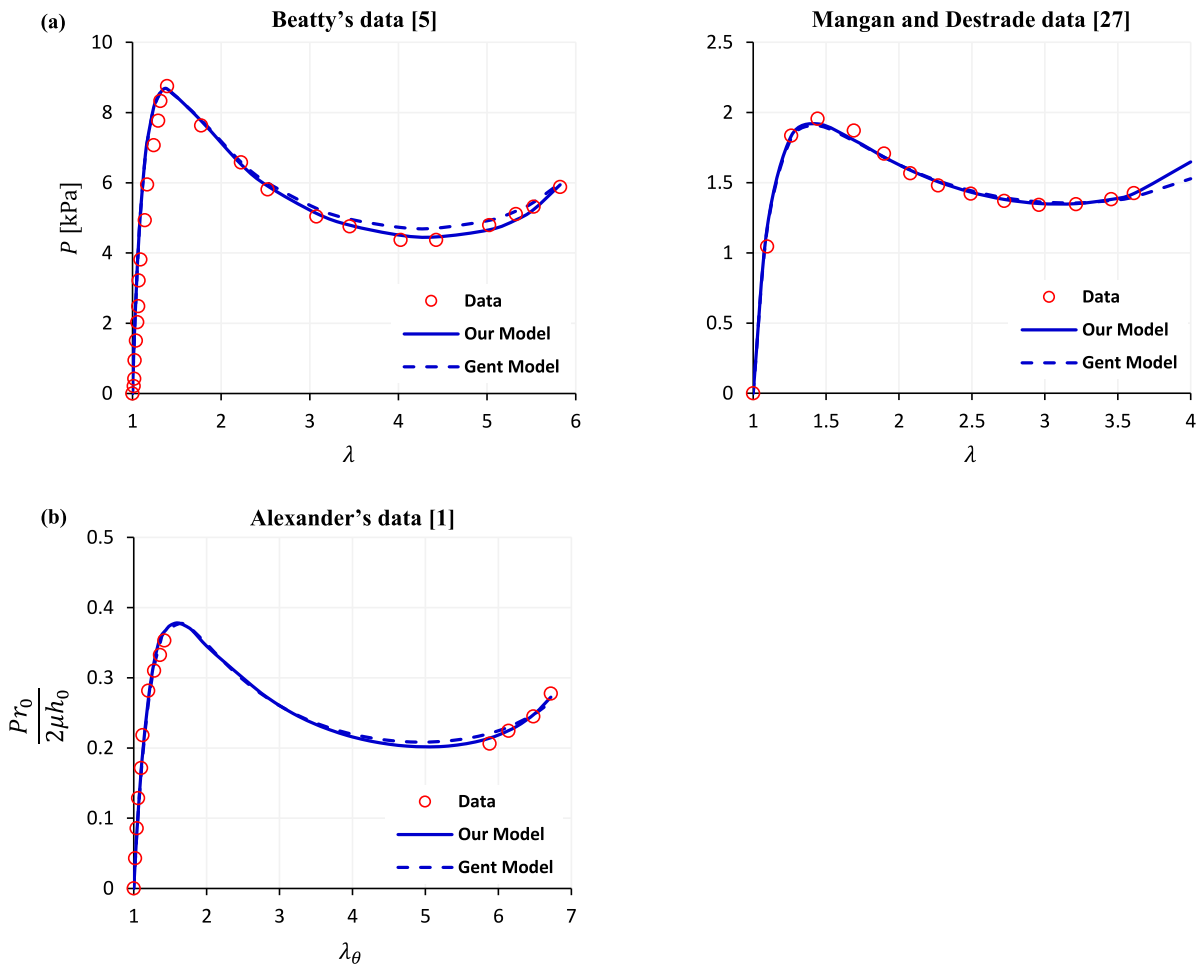

Fig. 18 Comparison between our proposed model and the Gent model for inflation of: (a) spherical; and (b) cylindrical thin shells. The continuous line represents our model and the dashed line is that of the Gent. The Gent model parameters for each dataset are: $\mu_{0}=345.6 \mathrm{kPa}, J_{m}=107.43$ for Beatty's data [5]; $\mu_{0}=58$ $\mathrm{kPa}, J_{m}=57.25$ for Mangan and Destrade data [27]; and $J_{m}=107.25$ for Alexander's data [1]

the cylindrical tube provided by [1], the $\mathrm{R}^{2}$ values of the fits provided by our model and the Gent model are 0.99 and 0.98 , respectively, while the RSS value of our model's fit is 0.002 and that of the Gent model is 0.003 . Therefore, again, while the differences in the fit goodness values are modest, our model provides an improved fit to the data compared with that of the Gent.

As noted in [19] and [22], a particular advantage of the Gent model is its mathematical simplicity, whereby with only two constitutive parameters it facilitates obtaining analytical solutions for a variety of boundary-value problems including torsion, shear and fracture deformation. Our proposed model, with a similar mathematical simplicity and two structurally relevant model parameters, appears to provide an even better description of the data and therefore ought to provide more accurate solutions for the forgoing considered boundaryvalue deformation problems.

We also note here, following Horgan and Saccomandi [20], that from a mathematical point of view the Gent model is a rational approximant of order [0/1] of the inverse Langevin function in terms of $I_{1}$. However, the model considered in this paper is a rational approximant of [1/1] order. Therefore, following the argument in [20], our model is a higher order constrained Padé approximation of the inverse Langevin function, preserving the original singularity point, so that both functions have the vertical asymptote at the same point. The singularity point may be viewed mechanistically as the point where the chain has reached 
its maximum allowable extension, beyond which failure may occur. This feature is an additional structural characteristic incorporated in our model, and may prove useful in future studies of investigating the failure point.

\subsection{A Note on the Addition of $I_{2}$ Terms}

As with all generalised neo-Hookean strain energy functions, our proposed model is limited to only $I_{1}$ terms. In this regard, the addition of an $I_{2}$ term may be judicious in order to provide even better-improved fits to the inflation data (note that for some modes of deformation such as shear, the inclusion of an $I_{2}$ term is necessary for capturing the true physics of the deformation - see, e.g., [9]). Without wishing to prompt the structural merits of one functional form over the other, and purely centred on a phenomenological basis at this point, we considered the addition of two types of $I_{2}$ terms commonly used in the literature: (i) a Mooney term in the form of $C_{2}\left(I_{2}-3\right)$; and (ii) a logarithmic term in the form of $C_{2} \ln \left(\frac{I_{2}}{3}\right)$ [32], so that:

$$
\left\{\begin{array}{l}
W_{i}=\mu N\left[\frac{1}{6 N}\left(I_{1}-3\right)-\ln \left(\frac{I_{1}-3 N}{3-3 N}\right)\right]+C_{2}\left(I_{2}-3\right), \\
W_{i i}=\mu N\left[\frac{1}{6 N}\left(I_{1}-3\right)-\ln \left(\frac{I_{1}-3 N}{3-3 N}\right)\right]+C_{2} \ln \left(\frac{I_{2}}{3}\right) .
\end{array}\right.
$$

We undertook fitting these two models to the inflation data of thin spherical balloons considered in $§ 3.1$. The pressure - inflation relationships may be derived using:

$$
P=4 \frac{h_{0}}{r_{0}}\left(\lambda^{-1}-\lambda^{-7}\right)\left(\frac{\partial W}{\partial I_{1}}+\lambda^{2} \frac{\partial W}{\partial I_{2}}\right) .
$$

Substituting the relationships in Eq. (36) into (37) we find:

$$
\left\{\begin{array}{l}
P_{i}=4 \frac{h_{0}}{r_{0}}\left(\lambda^{-1}-\lambda^{-7}\right)\left\{\mu\left[\frac{2 \lambda^{2}+\lambda^{-4}-9 N}{6\left(2 \lambda^{2}+\lambda^{-4}-3 N\right)}\right]+C_{2} \lambda^{2}\right\} \\
P_{i i}=4 \frac{h_{0}}{r_{0}}\left(\lambda^{-1}-\lambda^{-7}\right)\left\{\mu\left[\frac{2 \lambda^{2}+\lambda^{-4}-9 N}{6\left(2 \lambda^{2}+\lambda^{-4}-3 N\right)}\right]+\frac{C_{2}}{\lambda^{2}+2 \lambda^{-4}}\right\} .
\end{array}\right.
$$

Note that $I_{2}=\lambda^{4}+2 \lambda^{-2}$.

Interestingly, on using $W_{i}$, i.e., the model in Eq. (36) 1 containing a Mooney-type $I_{2}$ term, we did not find any noticeable improvement to the fittings compared with the $I_{1}$ only function $W$ in Eq. (1). Indeed, the value of $C_{2}$ was consistently close to zero. Using the $W_{i i}$ function, i.e., the model in Eq. (36) 2 containing the $C_{2} \ln \left(\frac{I_{2}}{3}\right)$ term also returned similar results, except for the dataset due to Rivlin and Saunders [34], where a significant improvement in the fitting was observed. In this case, the value of $C_{2}$ was found to be $C_{2}=0.14 \mathrm{MPa}$, with $\mu=0.24 \mathrm{MPa}$ and $N=21.79$. The fitting result is shown in Fig. 19 . The dashed line presents the model with the $C_{2} \ln \left(\frac{I_{2}}{3}\right)$ term; $P_{i i}$ in Eq. $(38)_{2}$, while the continuous line is due to the $I_{1}$-only function $W ; P$ in Eq. (8). The improvement in the fitting is evident, with $\mathrm{R}^{2}=0.99$ versus that of 0.97 by the $I_{1}$-only function. Also note the difference between the values of $N$, where $N=9.78 \times 10^{14}$ was characterised for this dataset using the $I_{1}$-only function $W$. Therefore, in this case, not only the logarithmic $I_{2}$ term has improved the fitting, it also appears to have captured the underlying physics more reasonably. 


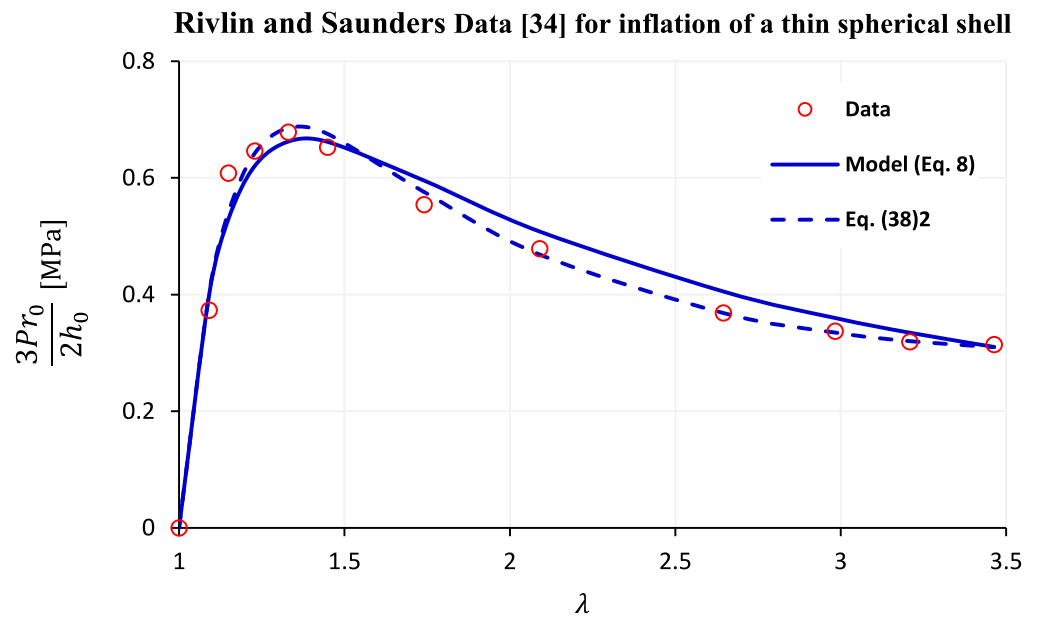

Fig. 19 Comparison between the $I_{1}$-only form of our proposed function $W$ (continuous line) and the addition of a logarithmic term in the form of $C_{2} \ln \left(\frac{I_{2}}{3}\right)$, shown with a dashed line, for the dataset due to [34]. Note the noticeable improvement in the fitting

Therefore, the addition of an $I_{2}$ term does not seem to automatically guarantee an improved fit in the case of inflation. Our proposed model, the $I_{1}$-only function $W$ in Eq. (1), appears to provide a very good fit to the majority of the considered datasets. The addition of a Mooney-type $I_{2}$ term did not facilitate an improvement to the fittings, and indeed the numerical value of $C_{2}$ was consistently close to zero. However, the addition of the $C_{2} \ln \left(\frac{I_{2}}{3}\right)$ term appears to be useful in better capturing the inflation data of some elastomer samples such as that due to Rivlin and Saunders [34].

\section{Concluding Remarks}

The new proposed model in Eq. (1) and the ensuing pressure - inflation relationships were shown to be crucially capable of capturing the true physics of inflation, including the limitpoint and inflation-jump instabilities. Of the four inflation cases of thick and thin shells considered and formulated here, it was shown that thick-walled spherical and cylindrical shells always exhibit a monotonic and stable inflation. The ascription of the limit-point instability (and by extension the ensuing inflation-jump) in the inflation of thin-walled spherical balloon and cylindrical tubes was, however, made to a critical value of the model parameter $N$, denoted here by $N_{t}$. For $N<N_{t}$, the inflation of thin-walled shells is predicted to be stable; however when $N>N_{t}$ the model predicts and captures the observed instabilities.

A wide range of experimental data using various techniques and over a three-century span of time were considered here to verify the application and capability of the proposed model. The overarching theme that emerged from all the fittings is that the proposed model generally provides a good description of the data, captures the observed instabilities and predicts maximum pressure values in good agreement with the experimental data. In addition to the rubber samples, two different datasets from the inflation of biological soft tissues were also considered. Soft tissues are known to possess low values of $N$, typically below those presented in Eqs. (12) and (27). When applied to the inflation data of those soft tissues, the model consistently characterised a low value of $N$, below that of $N_{t}$, and correctly predicted a stable inflation as was observed experimentally. The application of the model to all 
considered experimental datasets was made prima facie under the assumption that the shells maintain their cross-sectional profile during the inflation. The consistent agreement between the model and the experimental data for all the considered 12 independent datasets, however, provides a reassuring reliability on the accuracy and capability of the proposed model.

A comparison between the predictions of our proposed model versus those of the Gent model was also presented. It was shown that while the two models broadly behave in a similar manner, our model is capable of providing improved fittings to the data. This improvement was shown to be observed via the quality of fits, as well as in better $\mathrm{R}^{2}$ and RSS values. The addition of an $I_{2}$ term to the proposed functional form of the model was also considered, through a Mooney-type $C_{2}\left(I_{2}-3\right)$ as well as a Pucci-Saccomandi $C_{2} \ln \left(\frac{I_{2}}{3}\right)$ term. While it was shown that the addition of these $I_{2}$ terms did not automatically lead to an improved fitting compared to the proposed $I_{1}$-only function $W$ for most of the considered datasets, the $C_{2} \ln \left(\frac{I_{2}}{3}\right)$ term was capable of producing noticeable improvements in fittings to some of the datasets. Such an addition may therefore be recommended particularly in applications where the $I_{1}$-only function fails to provide an optimal fit.

As has been shown elsewhere, there are not many models in the literature that can capture the true physics of inflation and the pertaining instabilities. A famous quote attributed to George Box reminds us that "all models are wrong, but some are useful". Our analysis in this paper showed that the proposed generalised neo-Hookean model may be more useful than suspected. Thanks to Millard Beatty's driving research in the framework of nonlinear elasticity of rubbers and soft tissues, the proposed model provides more insight into better capturing of the "ultimate stiffening effect" and the ensuing instabilities. Given the simplicity of our model, sound theoretical background, favourable modelling results and the successful prediction of instabilities, we propose the application of this model to analysing and studying the inflation problem in rubber-like materials and soft tissues.

Acknowledgements The authors are grateful to Professor Michel Destrade for kindly providing us with the pressure - inflation data of spherical balloon samples and monkey bladder specimens used in [27]. Giuseppe Saccomandi is partially supported by PRIN project 2017KL4EF3_003 and GNFM of Istituto Nazionale di Alta Matematica (INDAM).

Publisher's Note Springer Nature remains neutral with regard to jurisdictional claims in published maps and institutional affiliations.

Open Access This article is licensed under a Creative Commons Attribution 4.0 International License, which permits use, sharing, adaptation, distribution and reproduction in any medium or format, as long as you give appropriate credit to the original author(s) and the source, provide a link to the Creative Commons licence, and indicate if changes were made. The images or other third party material in this article are included in the article's Creative Commons licence, unless indicated otherwise in a credit line to the material. If material is not included in the article's Creative Commons licence and your intended use is not permitted by statutory regulation or exceeds the permitted use, you will need to obtain permission directly from the copyright holder. To view a copy of this licence, visit http://creativecommons.org/licenses/by/4.0/.

\section{Appendix: Tabulated Experimental Data}

Table 3 Mallock (1891) data [26]

\begin{tabular}{ll}
\hline$v[-]$ & $P[\mathrm{kPa}]$ \\
\hline 1 & 0 \\
1.04 & 0.69 \\
$1.06_{5}$ & 1.38 \\
1.10 & 2.05 \\
\hline
\end{tabular}


A. Anssari-Benam et al.

Table 3 (Continued)

\begin{tabular}{ll}
\hline$v[-]$ & $P[\mathrm{kPa}]$ \\
\hline 1.13 & 2.57 \\
1.15 & 2.825 \\
1.20 & 3.60 \\
1.25 & 4.07 \\
1.27 & 4.22 \\
1.30 & 4.52 \\
1.35 & 4.81 \\
1.37 & 4.94 \\
1.405 & 5.11 \\
1.45 & 5.25 \\
1.47 & 5.31 \\
1.51 & 5.40 \\
1.55 & 5.48 \\
1.61 & 5.58 \\
1.65 & 5.62 \\
1.71 & 5.65 \\
1.76 & 5.67 \\
1.81 & 5.70 \\
1.86 & 5.65 \\
1.89 & 5.60 \\
\hline
\end{tabular}

Table 4 Beatty (1987) data [5]

\begin{tabular}{|c|c|}
\hline$\lambda[-]$ & $P[\mathrm{kPa}]$ \\
\hline 1 & 0 \\
\hline $1.01_{5}$ & 0.21 \\
\hline 1.02 & 0.42 \\
\hline 1.025 & $0.94_{5}$ \\
\hline 1.04 & $1.50_{5}$ \\
\hline $1.05_{5}$ & 2.03 \\
\hline $1.06_{5}$ & $2.48_{5}$ \\
\hline 1.07 & 3.22 \\
\hline 1.09 & $3.81_{5}$ \\
\hline 1.14 & 4.935 \\
\hline $1.16_{5}$ & 5.95 \\
\hline 1.24 & 7.07 \\
\hline 1.29 & 7.77 \\
\hline $1.31_{5}$ & 8.33 \\
\hline 1.39 & 8.75 \\
\hline $1.77_{5}$ & 7.63 \\
\hline 2.225 & 6.58 \\
\hline 2.525 & 5.81 \\
\hline 3.075 & 5.04 \\
\hline 3.45 & 4.76 \\
\hline 4.025 & 4.375 \\
\hline 4.425 & $4.37_{5}$ \\
\hline 5.025 & 4.80 \\
\hline 5.325 & 5.11 \\
\hline 5.525 & 5.32 \\
\hline 5.825 & 5.88 \\
\hline
\end{tabular}


Table 5 (Converted) Rivlin and Saunders (1951) data [34]

Table 6 Inflation data in [27] originally due to Merritt and Weinhaus (1978) [28]

Table 7 Data on the inflation of monkey bladder from [27], originally due to Osborne 1909 [31]

\begin{tabular}{ll}
\hline$\lambda[-]$ & $\frac{3 P r_{0}}{2 h_{0}}[\mathrm{MPa}]$ \\
\hline 1 & 0 \\
1.09 & 0.37 \\
1.15 & 0.61 \\
1.23 & 0.645 \\
1.33 & 0.68 \\
1.45 & 0.65 \\
1.74 & 0.55 \\
2.09 & 0.48 \\
2.645 & 0.37 \\
2.98 & 0.34 \\
3.21 & 0.32 \\
3.46 & 0.31 \\
\hline
\end{tabular}

\begin{tabular}{ll}
\hline$\lambda[-]$ & $P[\mathrm{kPa}]$ \\
\hline 1 & 0 \\
1.10 & $1.04_{6}$ \\
1.26 & $1.83_{5}$ \\
1.44 & $1.95_{5}$ \\
1.69 & 1.87 \\
1.90 & 1.71 \\
2.08 & 1.57 \\
2.27 & 1.48 \\
2.49 & 1.42 \\
2.72 & 1.37 \\
2.96 & 1.34 \\
3.21 & 1.35 \\
$3.45_{5}$ & 1.38 \\
3.61 & $1.42_{5}$ \\
\hline
\end{tabular}

\begin{tabular}{ll}
\hline$\lambda[-]$ & $P[\mathrm{kPa}]$ \\
\hline 1 & $0.09_{8}$ \\
1.10 & 0.14 \\
1.20 & 0.18 \\
1.30 & 0.22 \\
1.40 & 0.25 \\
1.50 & 0.29 \\
1.60 & 0.32 \\
1.70 & $0.39_{5}$ \\
1.80 & $0.44_{5}$ \\
1.90 & 0.54 \\
2.00 & 0.70 \\
2.10 & 0.93 \\
2.20 & 1.255 \\
2.22 & 1.31 \\
\hline
\end{tabular}


A. Anssari-Benam et al.

Table 8 Alexander (1971) data [1]

\begin{tabular}{ll}
\hline$\lambda_{\theta}[-]$ & $\frac{P r_{0}}{2 \mu h_{0}}[-]$ \\
\hline 1 & 0 \\
1.02 & 0.04 \\
1.04 & $0.08_{5}$ \\
1.06 & 0.13 \\
1.1 & 0.17 \\
1.12 & 0.22 \\
1.2 & 0.28 \\
1.28 & 0.31 \\
1.36 & 0.33 \\
1.42 & 0.35 \\
5.88 & 0.21 \\
6.14 & 0.22 \\
6.48 & 0.245 \\
6.72 & 0.28 \\
\hline
\end{tabular}

Table 9 Mallock (1891) data for inflation of cylindrical tubes [26]

\begin{tabular}{ll}
\hline$v[-]$ & $P[\mathrm{kPa}]$ \\
\hline 1 & 0 \\
1.03 & 1.76 \\
1.06 & 3.45 \\
1.10 & 5.285 \\
1.16 & 7.74 \\
1.20 & 9.19 \\
1.235 & 10.42 \\
1.275 & 11.64 \\
1.30 & 12.33 \\
1.34 & 13.18 \\
1.38 & 13.87 \\
1.40 & 14.325 \\
1.46 & 15.17 \\
1.50 & 15.78 \\
1.55 & 16.62 \\
1.60 & 17.31 \\
1.66 & 17.77 \\
1.70 & 18.00 \\
1.76 & 18.301 \\
1.80 & 18.46 \\
1.85 & 18.39 \\
1.91 & 18.23 \\
1.95 & 18.08 \\
2.00 & 17.62 \\
\hline & \\
\hline
\end{tabular}


Table 10 Charrier (1974) data [8]

Table 11 Holzapfel (2005) data for inflation of latex tubes [18]

Table 12 Holzapfel (2005) data for inflation of human iliac artery [18]

\begin{tabular}{|c|c|c|c|c|c|}
\hline \multicolumn{2}{|l|}{$\lambda_{z}=1$} & \multicolumn{2}{|c|}{$\lambda_{z}=1.5$} & \multicolumn{2}{|c|}{$\lambda_{z}=2.5$} \\
\hline$\lambda_{\theta}[-]$ & $\frac{P r_{0}}{h_{0}}[\mathrm{MPa}]$ & $\lambda_{\theta}[-]$ & $\frac{P r_{0}}{h_{0}}[\mathrm{MPa}]$ & $\lambda_{\theta}[-]$ & $\frac{P r_{0}}{h_{0}}[\mathrm{MPa}]$ \\
\hline 1 & 0 & 0.82 & 0 & 0.63 & 0 \\
\hline 1.04 & 0.07 & 0.85 & 0.07 & 0.66 & 0.07 \\
\hline 1.09 & $0.15_{5}$ & 0.90 & $0.14_{5}$ & 0.68 & 0.11 \\
\hline 1.15 & 0.30 & 0.93 & 0.28 & 0.71 & 0.15 \\
\hline \multirow[t]{3}{*}{1.27} & $0.45_{5}$ & 0.98 & 0.30 & 0.73 & 0.19 \\
\hline & & 1.08 & 0.38 & 0.76 & 0.28 \\
\hline & & & & 0.81 & 0.26 \\
\hline
\end{tabular}

\begin{tabular}{ll}
\hline$\lambda_{\theta}[-]$ & $P[\mathrm{kPa}]$ \\
\hline 1 & 0 \\
1.02 & 0.84 \\
$1.03_{5}$ & 1.27 \\
1.05 & 1.71 \\
1.06 & 2.18 \\
1.08 & 2.62 \\
1.09 & 3.09 \\
1.12 & 3.52 \\
1.14 & 3.92 \\
1.165 & 4.62 \\
1.20 & 5.05 \\
1.22 & 5.42 \\
1.25 & 5.63 \\
1.27 & 5.937 \\
1.30 & 6.18 \\
1.32 & 6.40 \\
\hline
\end{tabular}

\begin{tabular}{lc}
\hline$\lambda_{\theta}[-]$ & $P[\mathrm{kPa}]$ \\
\hline 1 & 0 \\
$1.00_{3}$ & 0.67 \\
$1.00_{5}$ & $1.04_{5}$ \\
$1.00_{7}$ & 1.94 \\
$1.01_{1}$ & 3.36 \\
$1.01_{4}$ & 6.27 \\
$1.01_{7}$ & 8.67 \\
$1.01_{9}$ & 11.64 \\
$1.02_{0}$ & 19.19 \\
$1.02_{1}$ & $26.86_{5}$ \\
$1.02_{2}$ & 33.94 \\
\hline
\end{tabular}


Table 13 Tube inflation data from the works of Kyriakides and Chang $[24,25]$

\begin{tabular}{|c|c|c|c|}
\hline \multicolumn{2}{|c|}{$\begin{array}{l}\text { Kyriakides and Chang } \\
\text { (1990) [24] }\end{array}$} & \multicolumn{2}{|c|}{$\begin{array}{l}\text { Kyriakides and Chang } \\
(1991)[25]\end{array}$} \\
\hline$v[-]$ & $\frac{1.1368 P r_{0}}{\mu h_{0}}[-]$ & $v[-]$ & $\frac{P}{\mu}[-]$ \\
\hline 1 & 0 & 1 & 0 \\
\hline 1.535 & 0.18 & 1.07 & 0.04 \\
\hline $2.78_{5}$ & 0.39 & 1.13 & 0.08 \\
\hline 3.14 & $0.67_{5}$ & 1.03 & 0.12 \\
\hline 3.68 & 0.82 & 1.47 & 0.16 \\
\hline 4.07 & 0.86 & 3.33 & 0.22 \\
\hline 8.14 & 0.73 & 8.00 & 0.18 \\
\hline 14.39 & 0.64 & 10.33 & 0.16 \\
\hline 24.21 & 0.55 & 16.67 & 0.14 \\
\hline 50.11 & $0.47_{5}$ & 20.33 & 0.13 \\
\hline 65.285 & 0.46 & 30.33 & 0.12 \\
\hline 100.019 & 0.46 & 40.67 & 0.11 \\
\hline 150.00 & 0.49 & 50.33 & $0.10_{9}$ \\
\hline 200.00 & 0.52 & 70.33 & $0.10_{8}$ \\
\hline 250.02 & 0.56 & 90.00 & 0.11 \\
\hline \multirow[t]{3}{*}{300.00} & 0.59 & 110.00 & 0.12 \\
\hline & & 130.33 & $0.12_{5}$ \\
\hline & & 149.67 & 0.13 \\
\hline
\end{tabular}

\section{References}

1. Alexander, H.: The tensile instability of an inflated cylindrical membrane as affected by an axial load. Int. J. Mech. Sci. 13, 87-95 (1971)

2. Anssari-Benam, A., Bucchi, A.: Modeling the deformation of the elastin network in the aortic valve. J. Biomech. Eng. 140, 011004 (2018). https://doi.org/10.1115/1.4037916

3. Anssari-Benam, A., Bucchi, A.: A generalised neo-Hookean strain energy function for application to the finite deformation of elastomers. Int. J. Non-Linear Mech. 128, 103626 (2021). https://doi.org/10.1016/ j.ijnonlinmec.2020.103626

4. Anssari-Benam, A., Pani, M., Bucchi, A.: Specialized strain energy functions for modeling the contribution of the collagen network $\left(\mathrm{W}_{\text {aniso }}\right)$ to the deformation of soft tissues. J. Appl. Mech. 87, 071006 (2020). https://doi.org/10.1115/1.4046894

5. Beatty, M.F.: Topics in finite elasticity: hyperelasticity of rubber, elastomers, and biological tissues - with examples. Appl. Mech. Rev. 40, 1699-1734 (1987)

6. Bucchi, A., Hearn, G.E.: Predictions of aneurysm formation in distensible tubes: Part A - theoretical background to alternative approaches. Int. J. Mech. Sci. 71, 1-20 (2013)

7. Bucchi, A., Hearn, G.E.: Predictions of aneurysm formation in distensible tubes: Part B - application and comparison of alternative approaches. Int. J. Mech. Sci. 70, 155-170 (2013)

8. Charrier, J-M.: Large elastic deformation theories and unstable deformations of rubber structures - thin tubes. Rubber Chem. Technol. 47, 788-802 (1974)

9. Destrade, M., Gilchrist, M.D., Murphy, J.G., Rashid, B., Saccomandi, G.: Extreme softness of brain matter in simple shear. Int. J. Non-Linear Mech. 75, 54-58 (2015)

10. Destrade, M., Saccomandi, G., Sgura, I.: Methodical fitting for mathematical models of rubber-like materials. Proc. R. Soc. A 473, 20160811 (2017). https://doi.org/10.1098/rspa.2016.0811

11. Dickie, R.A., Smith, T.L.: Viscoelastic properties of a rubber vulcanizate under large deformations in equal biaxial tension, pure shear, and simple tension. Trans. Soc. Rheol. 15, 91-110 (1971)

12. Gent, A.N.: A new constitutive relation for rubber. Rubber Chem. Technol. 69, 59-61 (1996)

13. Gent, A.N.: Elastic instabilities of inflated rubber shells. Rubber Chem. Technol. 72, 263-268 (1999)

14. Gent, A.N.: Elastic instabilities in rubber. Int. J. Non-Linear Mech. 40, 165-175 (2005) 
15. Gent, A.N., Thomas, A.G.: Forms for the stored (strain) energy function for vulcanized rubber. J. Polym. Sci. 28, 625-628 (1958)

16. Green, A.E., Adkins, J.E.: Large Elastic Deformations. Clarendon, Oxford (1960)

17. Green, A.E., Zerna, W.: Theoretical Elasticity. Clarendon, Oxford (1954)

18. Holzapfel, G.A.: Similarities between soft biological tissues and rubberlike materials. In: Austrell, P.E., Kari, L. (eds.) Constitutive Models for Rubber IV, Proceedings of the Fourth European Conference on "Constitutive Models for Rubber" ECCMR 2005, Stockholm, Sweden. Taylor \& Francis, London, pp. 607-617 (2005)

19. Horgan, C.O.: The remarkable Gent constitutive model for hyperelastic materials. Int. J. Non-Linear Mech. 68, 9-16 (2015)

20. Horgan, C.O., Saccomandi, G.: A molecular-statistical basis for the Gent constitutive model of rubber elasticity. J. Elast. 68, 167-176 (2002)

21. Horgan, C.O., Saccomandi, G.: A description of arterial wall mechanics using limiting chain extensibility constitutive models. Biomech. Model. Mechanobiol. 1, 251-266 (2003)

22. Horgan, C.O., Saccomandi, G.: Phenomenological hyperelastic strain-stiffening constitutive models for rubber. Rubber Chem. Technol. 79, 152-169 (2006)

23. Kanner, L.M., Horgan, C.O.: Elastic instabilities for strain-stiffening rubber-like spherical and cylindrical thin shells under inflation. Int. J. Non-Linear Mech. 42, 204-215 (2007)

24. Kyriakides, S., Chang, Y-C.: On the inflation of a long elastic tube in the presence of axial load. Int. J. Solids Struct. 26, 975-991 (1990)

25. Kyriakides, S., Chang, Y-C.: The initiation and propagation of a localized instability in an inflated elastic tube. Int. J. Solids Struct. 27, 1085-1111 (1991)

26. Mallock, A.: II. Note on the instability of India-rubber tubes and balloons when distended by fluid pressure. Proc. R. Soc. Lond. 49, 296-301 (1891)

27. Mangan, R., Destrade, M.: Gent models for the inflation of spherical balloons. Int. J. Non-Linear Mech. 68, 52-58 (2015)

28. Merritt, D.R., Weinhaus, F.: The pressure curve for a rubber balloon. Am. J. Phys. 46, 976-977 (1978)

29. Müller, I., Strehlow, P.: Rubber and Rubber Balloons - Paradigms of Thermodynamics. Springer, Berlin (2004)

30. Ogden, R.W., Saccomandi, G.: Introducing mesoscopic information into constitutive equations for arterial walls. Biomech. Model. Mechanobiol. 6, 333-344 (2007)

31. Osborne, W.A.: The elasticity of rubber balloons and hollow viscera. Proc. R. Soc. Lond. Ser. B 81, 485-499 (1909)

32. Pucci, E., Saccomandi, G.: A note on the Gent model for rubber-like materials. Rubber Chem. Technol. 75, 839-852 (2002)

33. Puglisi, G., Saccomandi, G.: The Gent model for rubber-like materials: an appraisal for an ingenious and simple idea. Int. J. Non-Linear Mech. 68, 17-24 (2015)

34. Rivlin, R.S., Saunders, D.W.: Large elastic deformations of isotropic materials VII. Experiments on the deformation of rubber. Philos. Trans. R. Soc. A 243, 251-288 (1951) 